\title{
DEVELOPMENT OF A HYPERSPECTRAL NIRS SYSTEM FOR FUNCTIONAL IMAGING OF THE BRAIN AND FOR CEREBRAL STATUS MONITORING DURING CARDIAC SURGERY
}

\author{
by \\ Ermias Woldemichael \\ Bachelor of Science, Ryerson University, 2014 \\ A thesis \\ presented to Ryerson University \\ in partial fulfillment of the requirements for the degree of \\ Master of Science \\ in the program of \\ Biomedical Physics
}

Toronto, Ontario, Canada, 2017

CErmias Woldemichael, 2017 
I hereby declare that I am the sole author of this thesis. This is a true copy of the thesis, including any required final revisions, as accepted by my examiners.

I authorize Ryerson University to lend this thesis to other institutions or individuals for the purpose of scholarly research.

I further authorize Ryerson University to reproduce this thesis by photocopying or by other means, in total or in part, at the request of other institutions or individuals for the purpose of scholarly research.

I understand that my thesis may be made electronically available to the public. 


\title{
Development of a Hyperspectral NIRS System
}

\section{for Functional Imaging of the Brain and for Cerebral Status Monitoring \\ during Cardiac Surgery}

\author{
Master of Science 2017 \\ Ermias Woldemichael \\ Biomedical Physics \\ Ryerson University
}

\begin{abstract}
Hyperspectral near infrared spectroscopy (hNIRS) is a noninvasive, real-time imaging modality with an improved quantitative accuracy and increased number of detectable chromophores. It uses the broadband spectrum of light wavelengths in the range of $700-$ $1100 \mathrm{~nm}$ and is based on the unique absorbance property of molecules and the fact that all biological tissues are relatively transparent to these wavelengths which allow for measuring concentrations of light absorbing molecules such as the Oxy- and Deoxy- hemoglobin and Cytochrome C Oxidase. As opposed to fMRI, PET and SPECT, hNIRS is inexpensive and portable.
\end{abstract}


The purpose of this thesis project was to employ advantages of hNIRS by developing the multichannel hNIRS set-up for the simultaneous assessment of multiple areas of the brain and to test the system in clinical applications. To achieve these goals, I developed a new optical fiber bundle design providing improvement of the optical power throughput into the hNIRS light detectors. I also developed a novel probe for measurements on hairy areas of the human head. To validate the hNIRS system I used it simultaneously with fMRI, which revealed a good correlation of hNIRS and fMRI BOLD signals from the brain. The multichannel hNIRS set up with the increased signals due to the novel optical fiber bundles was then used during various brain activation protocols, which in the future can allow for the assessment of patients with mild traumatic brain injuries (mTBI). Finally, the hNIRS system with the new fiber bundles was compared with a commercial NIRS system in clinical setting for brain monitoring of patients during the transcatheter aortic valve implantation operation (TAVI). 


\section{Acknowledgements}

First of all I would like to thank God for giving me the opportunity and the strength to go all the way to this moment. He could not have provided me with a better supervisor than Dr. Vladislav Toronov. My sincere gratitude to Dr. Toronov for the unparalleled patience and support he gave me through all the obstacles and challenges in the research. His immense knowledge and dedication towards this project during the research and writing this thesis has paved the way towards my master's degree.

I would like to deeply appreciate both members of my supervisory committee Dr. Raffi Karshafian and Dr. Nathan Churchill for their invaluable input and comments into my research progress. And my sincere gratitude to my entire research team for their patience and dedication during the countless trials in the lab and St. Michael's hospital. My special thanks to Dr. Toronov's family for their voluntary input to the success of the research.

Also, I would like to acknowledge professional and technical support I received from Ryerson University Department of Physics administration, technical officers and technologists. 


\section{Dedication}

To

My father, strong and gentle soul, for his love and encouragement and for teaching me to believe in hard work. 
Table of Contents

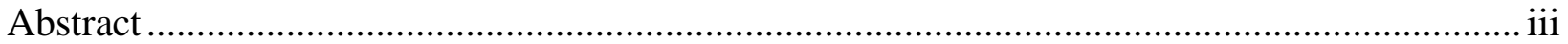

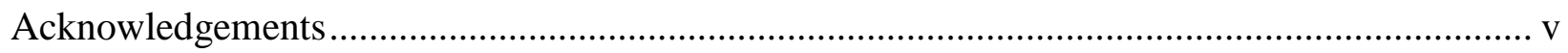

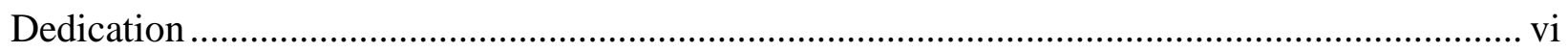

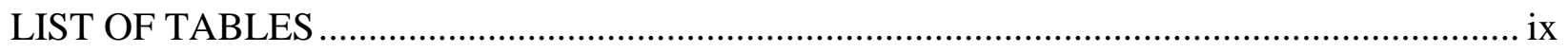

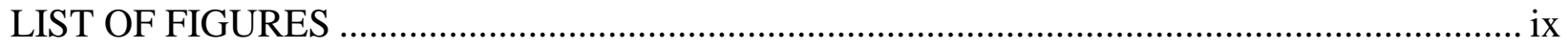

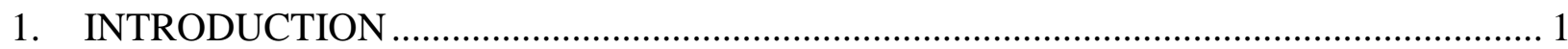

1.1 Functional near-infrared spectroscopy .......................................................... 1

1.2 Optical Properties of Biological Tissues ........................................................... 6

1.2.1 Modified Beer-Lambert Law .............................................................. 9

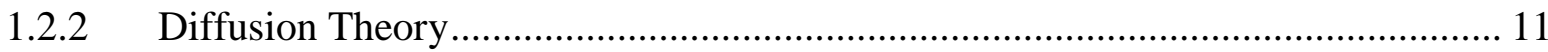

1.3. Functional Magnetic Resonance Imaging ......................................................... 14

1.4. Research Objectives and Hypothesis ........................................................... 16

2. DEVELOPMENT OF MULTICHANNEL hNIRS SYSTEM ...................................... 18

2.1. Development of Fiber Optic Bundles........................................................... 18

2.1.1. Fiber Optic Bundle measurement ............................................................... 20

2.2. Multichannel Setup and Measurements ................................................................ 21

2.2.1. Multichannel hNIRS Test .............................................................................. 24

2.3. Data Processing Algorithm ........................................................................... 25

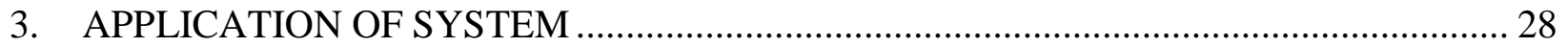




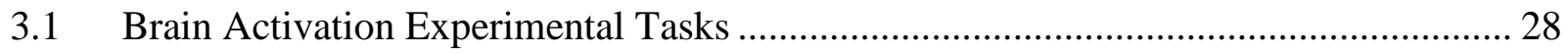

3.2 Brain Response to Cognitive Tasks as measured by hNIRS .................................... 29

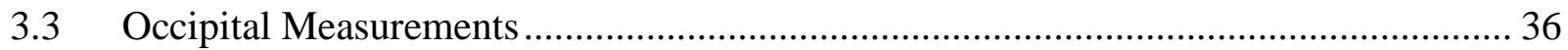

3.4 Measurements During Transcatheter Aortic Valve Implantation .............................. 42

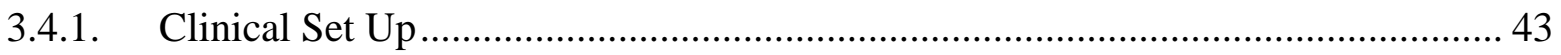

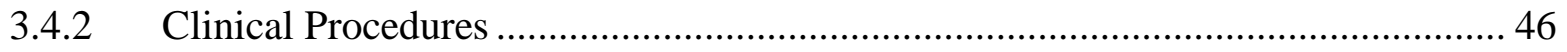

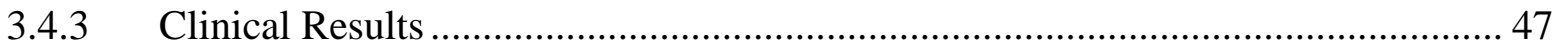

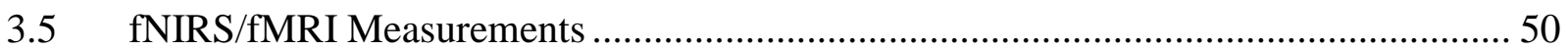

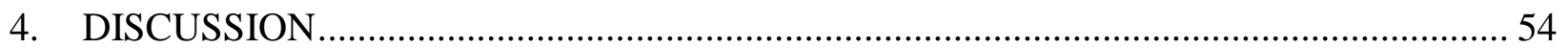

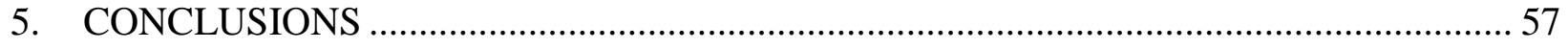

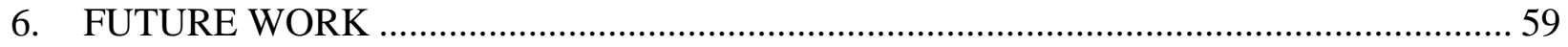

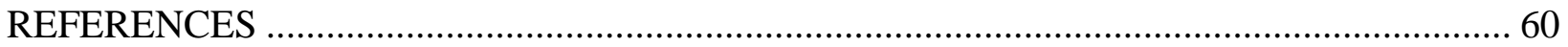




\section{LIST OF TABLES}

Table 1. T-test result in micromolars $(\mu \mathrm{M})$, with p-values in brackets, for the comparison between the Eyes Open and Eyes Closed regimes of all subjects (EC1, EO1 - first Eyes Closed, first Eyes Open, and EC2, EO2 - second Eyes Closed, second Eyes Open)........................... 33

Table 2. T-test result (in $\mu \mathrm{M}$ ) of the average difference between Rest period and Trails A and B, and between Trails A and B (p-values are indicated in brackets with $\mathrm{p}<0.05$ highlighted)...... 35 Table 3. T-test results in $\mu \mathrm{M}$, with p-values in brackets, for the Occipital comparison between the Eyes Open and Eyes Closed regimes of all subjects (EC1, EO1 - first Eyes Closed, first Eyes Open, and EC2, EO2 - second Eyes Closed, second Eyes Open).

\section{LIST OF FIGURES}

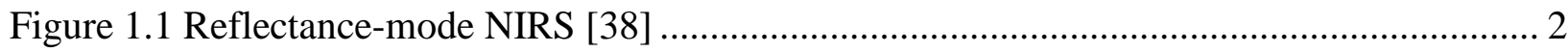

Figure 1.2 A Schematic showing the components of a spectrometer. .................................. 5

Figure 1.3 Absorption Spectra of main tissue chromophores in the near-infrared region of the

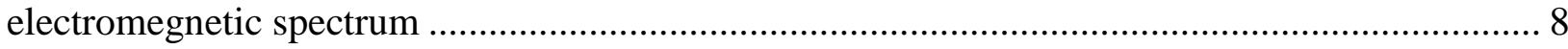

Figure 1.4 The point spread distribution of light in a tissue. ............................................. 9

Figure 2.1 a. The optic fiber bundle and, b. The linear arrangement of the fibers, c. The

numerical aperture (NA) of an optical system. 19

Figure 2.2 Comparison of fiber spectra. 20

Figure 2.3 a. The Source (Fiber-Lite® DC950H Machine Vision Fiber Optic Illuminator) and, b. The QE65000 scientific-grade spectrometer (Ocean Optics, Dunedin, Florida)..... 22 


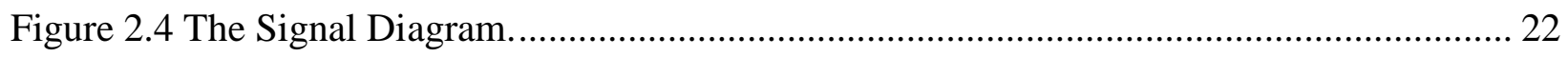

Figure 2.5 The Multi-Channel Experimental set up. ............................................................... 23

Figure 2.6 The Multichannel Raw data and b. Multichannel Raw Data Spectrum..................... 24

Figure 2.7 Multichannel SNR for IT at 500ms, 100s and average of five 100ms spectra............ 25

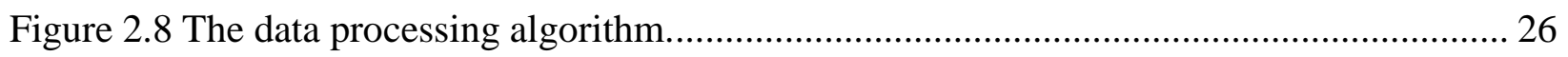

Figure 3.1 The neurocognitive task of Trail Making .............................................................. 29

Figure 3.2 The Experimental headset showing the hNIRS and the EEG probes.......................... 30

Figure 3.3 The electrode placement of the EEG (indicating F7 $(\mathrm{CH} 1)$ and F8 $(\mathrm{CH} 2)$ and

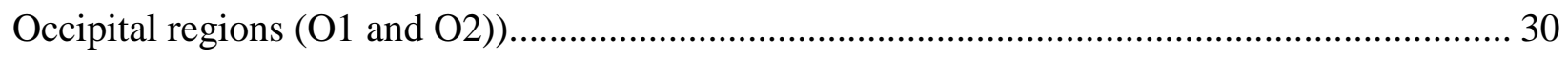

Figure 3.4 Averaged brain response to Eyes Open/Close task (Blue) and their corresponding standard deviation (Red) for a) Channel 1 and b) Channel 2. .................................................... 31

Figure 3.5 Averaged brain response (Oxy, Deoxy and Total) to Trail Making task (Blue) and their corresponding standard deviation (Red) for a) Channel 1 and b) Channel 2....................... 34

Figure 3.6 The experimental set up for Occipital measurement ................................................. 37

Figure 3.7 . a. Probe for Occipital measurement, and b. Optic fiber arrangement for the probe. 38

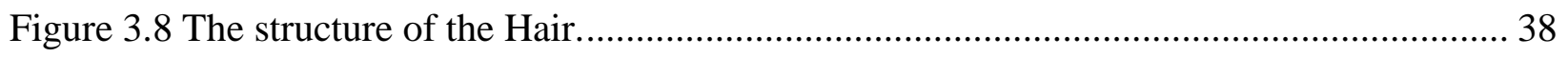

Figure 3.9 Spectra of the heads with Red and Black hair (integration time - 1 second)............... 39

Figure 3.10 fNIRS plots of all chromophores during the occipital measurement. ....................... 40

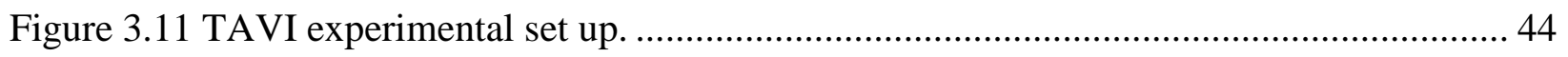

Figure 3.12 The TAVI measurement Probe.............................................................................. 44

Figure 3.13 The TAVI experimental set up including the C-Scan. ............................................. 46

Figure 3.14 Measurement of the Cerebral Oxygen Saturation. ................................................... 47 
Figure 3.15 Measurement of cerebral $\mathrm{Hb}$ and $\mathrm{HbO} 2$ during TAVI....................................... 48

Figure 3.16 . hNIRS versus Nonin measurement. ......................................................... 48

Figure 3.17 Patient 1: Comparison of SO2 measured by mNIRS and hNIRS. ........................ 49

Figure 3.18 Patient 1: Comparison of SO2 measured by mNIRS and Cyt-Ox measured by

hNIRS.

Figure 3.19 Patient 2: comparison of SO2 measured by mNIRS with Cyt-ox measured by hNIRS.

Figure 3.20 The experimental set up for an fNIRS/fMRI simultaneous measurement. The dashed line shows the separating wall between the MRI machine room and the adjacent room........... 51

Figure 3.21 fNIRS Vs Primary BOLD HRF............................................................ 52

Figure 3.22 Mean Z-scored BOLD activation maps for trail making task, comparing Task A+B

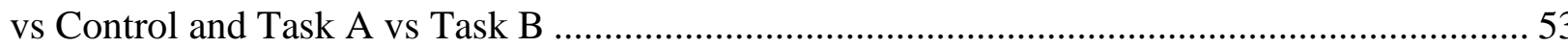




\section{INTRODUCTION}

\subsection{Functional near-infrared spectroscopy}

Near-infrared spectroscopy is a brain monitoring technique that uses the unique absorption characteristics of biological tissues to near-infrared (NIR) light (wavelength in the range between $600 \mathrm{~nm}-1100 \mathrm{~nm}$ ). Biological tissues are transparent to NIR light as each tissue chromophores (namely Hemoglobins and Cytochrome C Oxidase) have a unique low molar absorption in this range [1]. Research in functional near infra-red spectroscopy is motivated by its high potential as a real time and noninvasive method of monitoring brain hemodynamics.

As of today, various methods of measuring and imaging the brain functions, such as EEG, fMRI, PET, SPECT, have been developed. Among these, fMRI is most widely used to investigate cognitive processes [2] in the brain with best spatial resolution of $1 \mathrm{~mm}$ for humans and $0.1 \mathrm{~mm}$ for small animals. It uses the blood-oxygenation-level-dependent (BOLD) signal reflecting changes in the concentration of deoxyhemoglobin. However, fMRI is very expensive and requires settings that may be unsuitable for many clinical cases [2].

An alternative method that is a noninvasive, real-time measurement is functional nearinfrared spectroscopy (fNIRS), which is less expensive and relatively easy to use. fNIRS is based on the facts that all molecules have unique absorbance spectra and that biological tissues are relatively transparent to certain wavelengths of light. NIRS allows for measuring concentrations of the light absorbing molecules in the tissue (such as Oxy- and Deoxy- 
hemoglobin, water, and Cytochrome $\mathrm{C}$ Oxidase). In contrast to other brain imaging modalities fNIRS equipment is less expensive and much more portable.

In 1977 Jobsis [3] demonstrated the detection of the oxygenation of certain tissue molecules by the near-infrared light. Since 1990 NIRS began to be used in clinical research settings where the non-invasive and safe measurements were required. It has gained a significant recognition due to its continuous, real-time, and noninvasive nature. A novel approach was to utilize multimodality whereby NIRS was used with fMRI to complement and exploit the strengths and weaknesses of each method [4], [5].

If an infant's head is being tested the detector could be placed on the opposite side of the head from the source optode as infant head is transparent enough to allow light to pass through to the other side. In the case of adults this is not possible as the adult head is composed of different layers of tissues (scalp, skull, brain) and the light would be significantly attenuated by scattering and absorption from these layers. Therefore, in adults the reflectance-mode NIRS is used [10], [12] where the receiving optodes are placed ipsilateral to the transmitter at an approximate distance of 30mm (Figure 1.1).

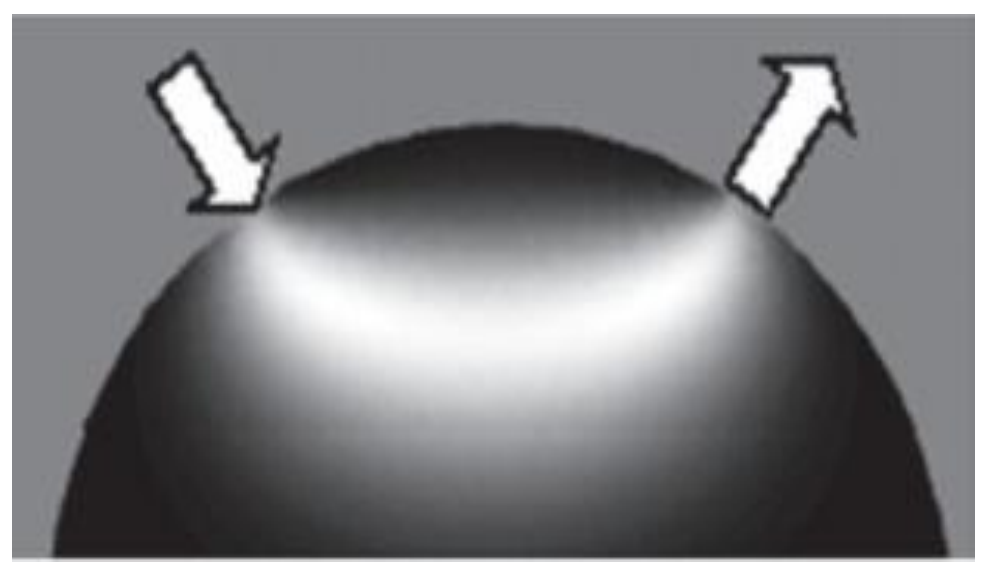

Figure 1.1 Reflectance-mode NIRS [38] 
Due to the light scattering by biological tissues, the path travelled by light in the tissue follows a diffuse pattern. Usually NIRS set-ups assume homogenous tissue that ensures an elliptical shape path in which the mean depth of penetration is proportional to the sourcedetector distance [10], [4]. With these assumptions the Modified Beer-Lambert Law (MBLL) calculates the chromophore concentration changes. But, as shown in section 1.2.1, MBLL is not completely accurate in biological media and therefore an alternative diffusion theory approach is discussed in section 1.2.2.

In recent time, several types of NIRS apparatuses were used to measure oxygenation changes in the brain. Bakker et al, [4], lists three different approaches in the measurement techniques of near infrared spectroscopes: Continuous Wave NIRS, Time-Resolved NIRS and Frequency Domain NIRS. The type of information one needs to collect basically determines the choice of the system. [4]

Continuous wave (CW) NIRS is the simplest and most widely used [13], [14] to resolve changes in $\mathrm{Hb}$ and $\mathrm{HbO} 2$ concentrations in the brain. However, $\mathrm{CW}$ NIRS is unable to quantify the absolute concentration of $\mathrm{Hb}$ and $\mathrm{HbO} 2$ as it cannot determine the optical path length [4], [13], [14]. CW NIRS approaches include the use of simple Modifies BeerLambert Law (MBLL) to calculate changes in concentrations from the changes in the light attenuation based on simplified assumptions of homogeneous medium and that change in concentrations of the chromophores is the only cause of the attenuation [4]. It is with the development of multichannel CW NIRS, which used multispectral sources, that functional NIRS (fNIRS) became possible. 
Time-Resolved NIRS (TR NIRS) can be used to achieve the quantification of the absolute concentrations of the chromophores by using picoseconds laser pulses [15]. TR NIRS provides measures of both absorption and scattering properties of the tissue by employing the shape of the temporal photon distributions upon exiting the tissue $[14,15]$. The acquired distribution is analyzed by fitting the temporal profile with the diffusion theory model [4], [14] (described in section 1.2.2.). TR NIRS systems are costly and suffer low signal-to-noise ratio (SNR).

Frequency-Domain (FD) NIRS, which is, mathematically, the Fourier transform of the TR NIRS, measure the intensity, phase and modulation changes [4] by using amplitudemodulated radio frequency light [15]. FD NIRS measures both absorption and scattering of the medium by using the amplitude attenuation and phase shift of the detected signal with respect to the sinusoidally modulated incident laser signal [15]. Similar to TR NIRS, FD NIRS is noisy and relatively expensive compared to CW NIRS (but less expensive than TR NIRS). Looking at the strengths and weaknesses of each technique [15] one can choose the type of NIRS based on which kind of measurement is required. Since for fNIRS the main focus is on measuring changes in chromophore concentrations rather than on absolute measurements, CW NIRS is most suitable. The advantages of the CW NIRS are that it provides best SNR of all types of NIRS, it is most portable, and least expensive [15].

Hyperspectral (broadband) NIRS provides significant specificity and accuracy advantages compared to multispectral fNIRS. As opposed to multispectral NIRS, which typically uses from two to four discrete wavelengths, broadband or hyperspectral NIRS uses wavelengths between 650 and $1000 \mathrm{~nm}$ acquired at spectral resolutions close to $1 \mathrm{~nm}$. Both frequency-domain (FD) and time-resolved (TR) [4] NIRS can separate the scattering and 
absorption properties of tissues but have disadvantages of being noisier and more expensive compared to continuous-wave (CW) NIRS [15]. CW NIRS uses multispectral or hyperspectral wavebands. hNIRS setups include broadband light sources and spectrometers with CCD detectors.

Spectrometer Components:

1. SMA Connector

2. Slit

3. Filter

4. Collimating Mirror

5. Grating

6. Focusing Mirror

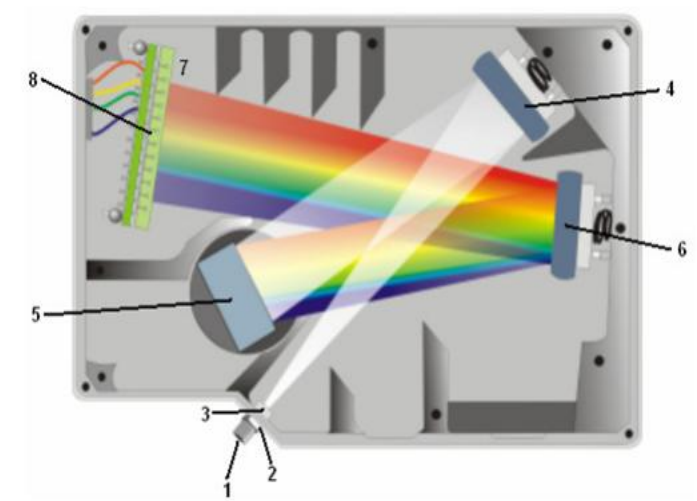

7. L2 Detector Collection Lens

8. CCD Detector

Figure 1.2 A Schematic showing the components of a spectrometer.

Figure 1.2 shows the schematic of a portable fiber-optic spectrometer, where different optical parts of the spectrometer that are arranged in such a way that the end result is a welldefined spectra of the sample to be examined.

The feasibility of hNIRS in tissue measurements was previously demonstrated by measuring concentrations of the chromophores in phantoms [8] and in-vivo [7], [9]. Yeganeh et al [7] developed hNIRS method to measure concentrations of deoxyhemoglobin ([Hb]), oxyhemoglobin ([HbO2]) and water of the brain tissue of piglets which were found close to those measured by frequency-domain NIRS. 


\subsection{Optical Properties of Biological Tissues}

Blood is a tissue that comprises many components, such as plasma, white and red blood cells and blood platelets. These components include a few chromophores that absorb light strongly in the near-infrared range $(\sim 650-1150 \mathrm{~nm}[10])$. Chromophores are molecular groups that are responsible for light absorption and express the color of the molecule [16]. The essential chromophores of importance for these studies are Deoxyhemoglobin $(\mathrm{Hb})$, Oxyhemoglobin ( $\mathrm{HbO} 2)$ and Cytochrome $\mathrm{C}$ Oxidase (CCO) as they are dominant on the near-infrared wavelengths and are biologically relevant markers for brain activity [15]. The absorption of the NIR light by $\mathrm{Hb}, \mathrm{HbO} 2$, and Cytochrome C Oxidase allows for measuring physiological parameters such as tissue oxygenation and oxygen metabolism rate. Several studies have been conducted to show hemodynamic responses in tissue by estimating changes in the concentrations of $\mathrm{Hb}$ and $\mathrm{HbO} 2$ and the total blood volume, which is proportional to addition of $[\mathrm{Hb}]$ and $[\mathrm{HbO} 2]$ ([] denote the concentrations of the chromophores) [13].

Hemoglobin is an iron-containing compound that is found in the red blood cell and gives it its red color. Hemoglobin consists of globulin chains (protein molecules) that also contains heme compound (a porphyrin compound). Each heme has a Fe center that is crucial for the oxygen transportation by the blood [17]. Depending on whether the oxygen molecule is attached to the hemoglobin, it occurs in two forms $-\mathrm{Hb}$ and $\mathrm{HbO}$. NIR specific absorption of these forms are different (see Figure 1.3), where the $\mathrm{HbO} 2$ peaks around $950 \mathrm{~nm}$ and the $\mathrm{Hb}$ at 760nm [10]. Changes in the concentration $[\mathrm{Hb}]$ and [HbO2] dominate the absorption of 
light in tissues [15] and are very important for the studies of the physiological changes during brain activity.

Studies conducted on the sensory stimulation and cognitive task responses [18], [13] suggest that during brain activity there is a significant increase in [HbO2] and a decrease in $[\mathrm{Hb}]$ in specific brain areas.

Cytochrome $\mathrm{C}$ Oxidase $(\mathrm{CCO})$ is an enzyme that is found in the mitochondrial membrane. It is the last enzyme in the respiratory electron transport chain and is responsible for the majority of the consumption of oxygen [17] and participates in synthesizing ATP. Adenosine triphosphate (ATP) is a nucleoside triphosphate used for intracellular energy transfer. This energy is used by cells for metabolism. It contains three phosphate groups [19]. Cells use enzymes to produce ATP by a metabolic process called phosphorylation. This process, which occurs in the mitochondrial membrane, generates most of the ATP needed in the cell. ATP is produced by energy released by redox reactions when electrons are transferred between donors and acceptors [17], [20]. Energy is released from ATP when it breaks down into ADP and a phosphate group.

One of the four redox-active centers of the $\mathrm{CCO}$ enzyme is a copper atom that absorbs light strongly in the near-infrared region. The NIR absorption of CCO peaks between $820-$ 840nm [10] (Figure 1.3.). The CCO concentration is less than $10 \%$ of the $[\mathrm{Hb}]$ and has a specific spectral signature [21]. 


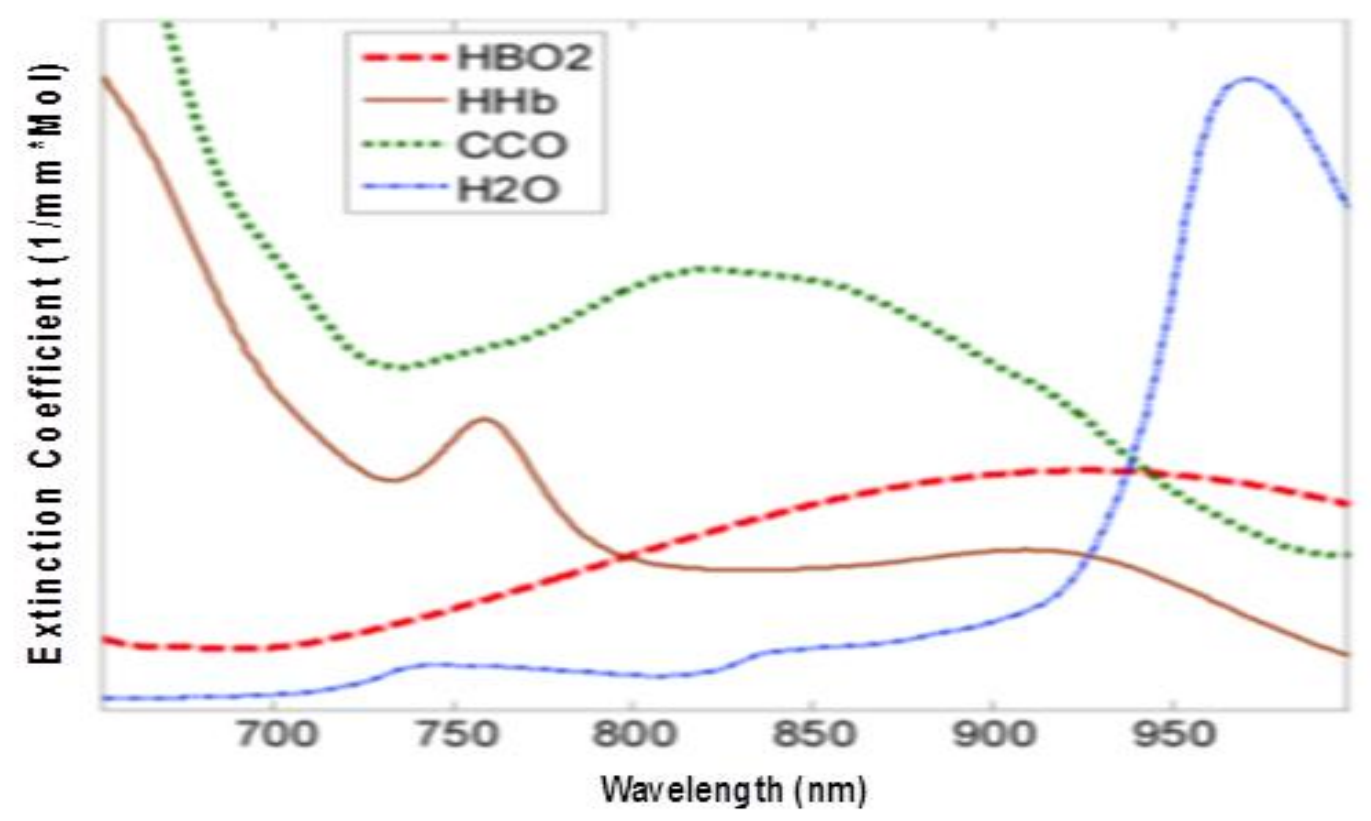

Figure 1.3 Absorption Spectra of main tissue chromophores in the near-infrared region of the electromegnetic spectrum

Figure 1.3 shows the specific absorption (extinction) coefficients of $\mathrm{Hb}, \mathrm{HbO} 2$, and $\mathrm{CCO}$. The extinction coefficient of a molecule is the specific absorbance by one mole of the molecule per one mm and is measured in micromoles per millimeter $\left(\mu \mathrm{Mol}^{-1} \mathrm{~mm}^{-1}\right)$.

Although the molecules in the biological tissues have characteristic and unique absorbance properties that make them detectable using NIR light, there are several factors that affect the measurements of these molecules in the tissues. Attenuation of light occurs not only due to the absorption, but also due to the scattering. As tissues are heterogeneous in nature and have varying refractive indices, scattering will occur when light passes through the different components of the tissue [11]. Also, due to the multiple scattering many photons will escape without being detected / absorbed. These optical properties are modeled using different approaches, such as MBLL and the diffusion approximation. 


\subsubsection{Modified Beer-Lambert Law}

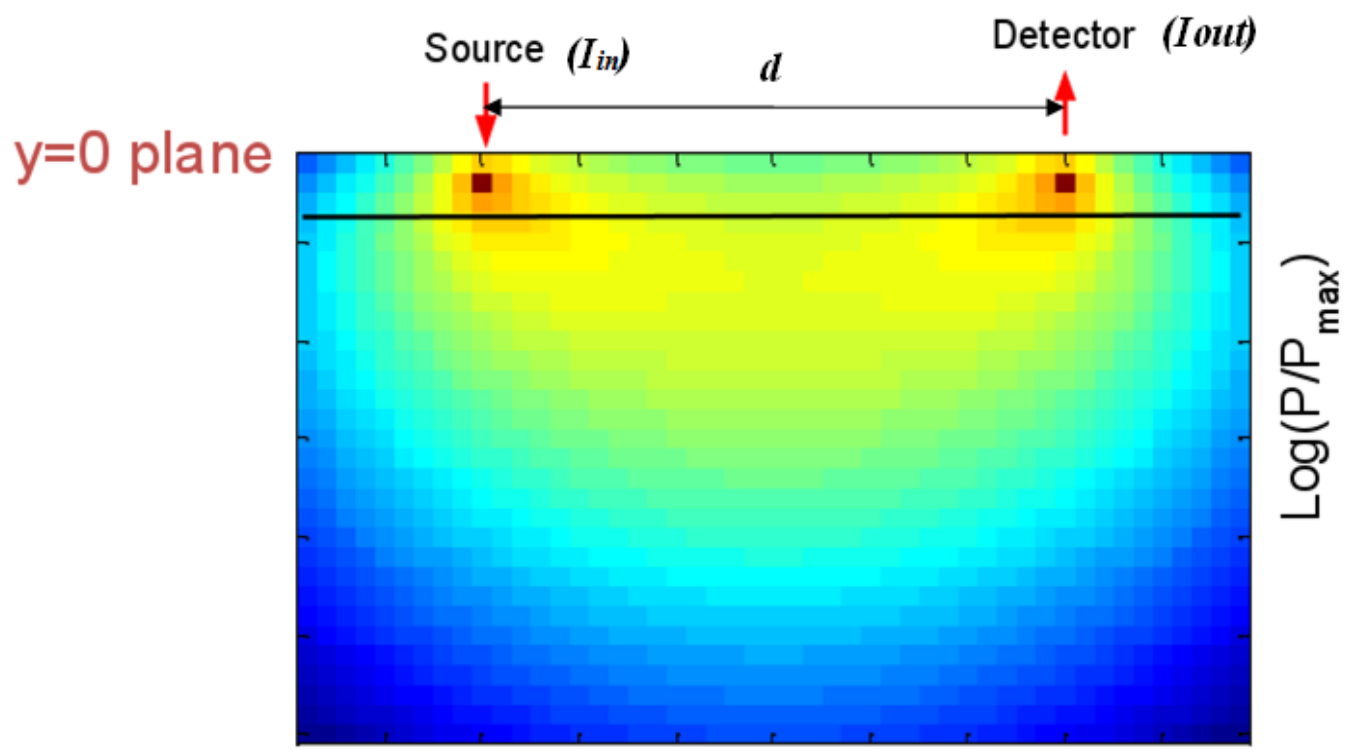

Figure 1.4 The point spread distribution of light in a tissue.

The Beer-Lambert Law calculates the chromophore concentrations by equating the decrease in light intensity as it transmits through a tissue and the extinction coefficient of the chromophore. The Beer-Lambert Law assumes that light will travel in a straight line in a medium. It combines Beer's and Lambert's Laws. The Beer's Law [26] relates the absorption of light in a medium (A) to the chromophore concentration (C) with the equation:

$$
A=\log \frac{I_{\text {in }}}{I_{\text {out }}}=\epsilon(\lambda) C L
$$

where $I_{\text {out }}$ is the intensity of the light transmitted after the light passes through the medium, $I_{\text {in }}$ is the intensity of the incident light, $\epsilon(\lambda)$ is the specific extinction coefficient of wavelength $\lambda, C$ is the chromophore concentration, and $L$ is the path length travelled by the light in the medium. The extinction coefficient $\epsilon(\lambda)$ of a molecule is the specific absorbance 
by 1 mole of the molecule per $1 \mathrm{~cm}$. And Lambert's Law [26] relates the light pathlength in a medium to the light absorbance by the equation:

$$
A=\mu_{a} d
$$

where $\mu_{a}$ is the absorption coefficient (in $\mathrm{mm}^{-1}$ ) and $d$ is the thickness of the medium. The absorption coefficient is the probability of photon absorption by a medium per unit pathlength and is give by:

$$
\mu_{a}=[H b] \varepsilon(\lambda)_{H b}+[H b O 2] \varepsilon(\lambda)_{H b O 2}+[C C O] \varepsilon(\lambda)_{c c o}
$$

The Modified Beer-Lambert Law (MBLL) [26] is adopted from the Beer-Lambert Law to include the fact that light scatters inside tissues. It can be used to quantify the absorption changes in the concentration of these chromophores. It relates the differences between the source and detector intensities with the concentration of the chromophores in the tissue delivered at specific wavelengths. And due to the medium being highly scattering (biological tissues), the MBLL accounts for the increase in pathlength of the light in the medium and the loss of photons due to the scattering. It is generally given by:

$$
A=\epsilon(\lambda) \operatorname{CdDPF}(\lambda)+G(\lambda)
$$

where $\operatorname{DPF}(\lambda)$ is the differential pathlength factor, and $G(\lambda)$ is the scattering loss factor. Only changes in absorption and chromophore concentration are possible as the $G$ value is unknown [22]. To account for the photon loss due to scatter (G), the Modified Beer-Lambert Law assumes that the change in scatter is negligible, and, hence, that the differential pathlength factor $(D P F)$ is constant [23], [22]. It also assumes that the absorption change of the medium is homogeneous. Hence, the Modified Beer-Lambert law can be written as 


$$
\Delta A=\epsilon(\lambda) \triangle C d D P F(\lambda)
$$

The accuracy of the Modified Beer Lambert Law is questioned due to the unrealistic assumptions it made on the homogeneity of the absorption changes and the scattering losses are constant. These assumptions do not hold true due to the fact that in reality tissue absorption changes are spatially inhomogeneous and that the absorption change is affected due to cross-talk between chromophores [24]. At the NIR range there is high scattering phenomenon due to photon-tissue interaction [23] which affects the differential pathlength factor (DPF) in the above MBLL equations. Hence, the photons follow an elliptical path that is a distribution of paths (Figure 1.4.). Other factors that limit the near-infrared spectroscope include wavelength, tissue type, blood volume and optode geometry.

\subsubsection{Diffusion Theory}

Since biological tissue comprises of many layers of scattering media, it is a highly scattering heterogeneous medium. Light intensity is lost as light passes through each scattering media in the tissue. This unknown loss of light intensity due to scattering in the medium is the main hindrance in predicting the path of the light in tissues. Some of the models used to overcome this obstacle use the Monte Carlo method that can be applied to inhomogeneous media but requires considerable computation time. The radiative transfer equation (RTE) is used to model the photon transport in biological tissue and it can be approximated to a diffusion equation [25]. Several methods were employed to find the solution of the diffusion equation to model absorbance. 
Before we look into the diffusion equation, we will first define some of the terms that are found in the equation. The scattering coefficient $\mu_{s}$ is wavelength dependent and describes the photon scattering per unit length [25]. It is the reciprocal of the mean free path and has a unit of $\left[\mathrm{mm}^{-1}\right]$ :

$$
\mu_{s}=\frac{1}{\text { meanfree path }}
$$

The reduced scattering coefficient $\left(\mu_{s}{ }^{\prime}\right)$ is defined as the probability of equivalent isotropic photon scattering by a medium per unit pathlength in diffusive regime [25] and is given by the equation

$$
\mu_{s}^{\prime}=\mu_{s}(1-g)
$$

where $g$ is the anisotropy factor of scattering which describes the average of cosine of scattering polar angle by single scattering and depends on the incident angle and wavelength of the source. It has a value between -1 and 1 . When $g=0$ the scattering is isotropic and when $g \approx 1$ we have a dominant forward scattering [25].

Assuming that we have a high scattering medium (which the biological medium is) where the absorption coefficient is much smaller than the scattering coefficient $\left(\mu_{a} \ll \mu_{s}{ }^{\prime}\right)$ and that the mean free path of the light is significantly shorter than the minimum thickness of the medium [26], the diffusion approximation can be given as [13] :

$$
-D(r) \nabla^{2} \Phi(r, t)+v \mu_{a} \Phi(r, t)+\frac{\partial \Phi(r, t)}{\partial t}=v S(r, t)
$$

where $D(r)$ is the diffusion coefficient, $r$ is position, $\Phi(r, t)$ is the photon intensity, $t$ is the time, $S(r, t)$ is the source and $v$ is the speed of light. The diffusion coefficient, $\mathrm{D}$, is given by $D=v / 3 \mu_{s^{\prime}}$ where $\mu_{s}{ }^{\prime}$ is the reduced scattering coefficient. 
Due to the positions of the source and the detector fibers on the tissue surface, the homogenous semi-infinite medium is more appropriate to model the absorption with diffusion equation. And the solution to the diffusion equation for a semi-infinite medium is given by [27]:

$\psi=\frac{2}{(4 \pi)^{2}} \frac{S}{D} \frac{\exp \left[-\rho\left(\frac{\mu_{a}}{D}\right)^{\frac{1}{2}}\right]}{\rho^{3}}\left[1+\rho\left(\frac{\mu_{a}}{D}\right)^{1 / 2}\right]\left(z_{0}+z_{b}\right) \times\left[z_{b}+3 D\left[1-\frac{\left(z_{0}+z_{b}\right)^{2}+3 z_{b}{ }^{2}}{2 \rho^{2}}\left\{3+\frac{\rho^{2} \frac{\mu_{a}}{D}}{1+\rho\left(\frac{\mu_{a}}{D}\right)^{1 / 2}}\right\}\right]\right]$

where $D=\frac{1}{3\left(\mu_{a}+\mu_{s}^{\prime}\right)}$ is the diffusion coefficient, the reduced scattering coefficient $\left(\mu_{s}{ }^{\prime}=(1-g) \mu_{s}, \rho\right.$ is the source-detector distance $(\mathrm{cm}), \mathrm{S}$ is the source strength (W), $z_{0}=3 D$ is the depth at which all photons are scattered and $z_{b}=2 \times D \times n_{r e l}\left(n_{r e l}\right.$ is the relative index of refraction) is the distance between extrapolated boundary and physical surface. The reduced scattering coefficient $\mu_{s}{ }^{\prime}$ at $800 \mathrm{~nm}$ wavelength can be given by

$$
\mu_{s}^{\prime}(\lambda)=\mu_{s 800}{ }^{\prime}\left(\frac{\lambda}{800}\right)^{-\alpha}
$$

where $\mu_{s 800}$ 'is the reduced scattering coefficient at the isobestic point $(\lambda=800 \mathrm{~nm})$. The value of $\alpha$ and $\mu_{s^{\prime} 800}$ for different tissue types were experimentally measured by Yeganeh et al [7].

Solving the diffusion equation analytically was limited to homogeneous medium and certain simple geometries. Using finite-element method to solve the diffusion equation is advantageous for its speed and can be used for inhomogeneous media [27]. The diffusion approach is an alternative to the Modified Beer-Lambert Law. 


\subsection{Functional Magnetic Resonance Imaging}

The physiological nature of the functional Near Infrared Spectroscopy (fNIRS) and Magnetic Resonance Imaging (MRI) are similar as signals measured by both modalities are correlated with the Hemoglobin response when a brain region is activated [13], [22], [5]. fMRI provides high resolution 3D images of the oxygenation -related changes in the magnetic susceptibility of brain tissue during functional activations. fMRI uses the bloodoxygenation-level-dependent (BOLD) signal to measure the concentration of Deoxyhemoglobin. The BOLD contrast based on the $\mathrm{Hb}$ and $\mathrm{HbO} 2$ magnetic susceptibility differences affecting the radiofrequency magnetic resonance signal. That is, these two forms of Hemoglobin have different magnetic properties $(\mathrm{HbO} 2$ is diamagnetic and $\mathrm{Hb}$ is paramagnetic). When a certain region of the brain is activated, a modest increase in the cerebral metabolic rate of oxygen is accompanied by a much larger increase in local blood flow. During this activation, $[\mathrm{Hb}]$ is decreased in comparison with the resting condition which is the basis for the BOLD effect.

The Gradient Echo (GE) technique for BOLD fMRI study uses the paramagnetic susceptibility of the $\mathrm{Hb}$ to measure the blood oxygenation changes [28]. The $\mathrm{Hb}$ molecules in the blood create a difference in magnetic susceptibility between the blood vessel and the surrounding tissue which is "felt" by the water molecules both in the blood and the surrounding tissue [29]. This local magnetic field gradient enhances the dephasing of water protons in the tissue [30] and reduces the signal intensity so that the image voxel appears darker [29]. MRI techniques that are based on the tissue oxygenation state (which influence 
the tissue-relaxation parameters $\mathrm{T} 1$ and $\mathrm{T} 2$ ) can be used for high spatial-resolution brain activity imaging [28].

These two independent tissue relaxation processes (transverse and longitudinal relaxation) dampen the received signal. The transverse relaxation is caused by the dephasing of spins relative to other nearby spins and the longitudinal relaxation is a process that is concerned with the longitudinal magnetization. T2 and T1 are the time constants of the two decays (transverse and longitudinal relaxation respectively). The transverse relaxation is represented as

$$
M_{x y}=M_{o} \sin \alpha e^{-j\left(2 \pi v_{o} t-\phi\right)} e^{-t / T_{2}}
$$

where $M_{x y}$ is the transverse magnetization, $\phi$ is the phase angle, $v_{o}$ is the Larmor frequency. And the longitudinal magnetization obeys:

$$
M_{z}=M_{0}\left(1-e^{-\frac{t}{T_{1}}}\right)+M_{Z}\left(O^{+}\right) e^{-t / T_{1}}
$$

where $M_{z}\left(O^{+}\right)=M_{0} \cos \alpha$ is the longitudinal magnetization immediately after the $\alpha$-pulse [31].

Proton density, T1 and T2 relaxation times determine the signal intensity on the MR image [32]. This signal in MR image changes depending on the pulse sequence used. In the gradient echo technique the signal strength decays with time constant $\mathrm{T}_{2}{ }^{*}$ (which satisfies $\mathrm{T}_{2}{ }^{*}$ $<\mathrm{T}_{2}$, the received signal decays faster than $\mathrm{T}_{2}$ ) as it refocuses the phase deviations [31]. This is caused by local perturbations in the static magnetic field $\mathrm{B}_{0}$ and can be related by the equation: 


$$
\frac{1}{T_{2}^{*}}=\frac{1}{T_{2}}+\frac{1}{T_{2}^{\prime}}
$$

In a $T_{2}$-weighed image (which uses longer repetition time (TR) and echo time (TE)) the CSF appears brighter [31].

An increase in the BOLD signal indicates a decrease in deoxyhemoglobin concentration $([\mathrm{Hb}])$ in the activated area as shown by many studies [33], [5], [34]. These studies have shown a good correlation between NIRS and MRI (BOLD) measurements.

\subsection{Research Objectives and Hypothesis}

The aim of my project was to develop and test a multichannel hyperspectral fNIRS system. Hyperspectral (broadband) NIRS uses thousands of near-infrared wavelengths, provides significantly better specificity and accuracy compared to multispectral the fNIRS, which typically uses 2-5 selected wavelengths. Potentially hNIRS can be used to measure both cerebral hemodynamic responses and changes in oxygen consumption by neurons during functional activations. However, due to specific features of spectrometers used as the detectors, hNIRS requires an improvement of the optical power throughput into the detectors and the development of the multichannel configuration, which is necessary for the simultaneous assessment of multiple areas of the human brain.

Furthermore, in this research project the hNIRS system was used simultaneously with fMRI. As the fMRI uses the BOLD (Blood Oxygenation Level Dependent) signal to detect brain activity changes during several cognitive activities, it can validate the results of the hNIRS data and allow us to develop hNIRS without fMRI. In future hNIRS may be useful 
for assessments of brain health of various types of patients with brain health concerns from mild traumatic brain injury to the cardiac arrest.

My hypotheses are:

1) Our custom-made fiber bundles will provide an increased signal as opposed to the available commercial fibers and be well fit to various settings.

2) Our multichannel set up will allow for measuring cerebral tissue chromophores on multiple brain regions.

3) Simultaneous hNIRS and fMRI data collected with these fibres will be of good quality and correlate well.

4) The hNIRS measurements made with these set-ups during clinical applications should provide applicable and meaningful results compared to other modalities. 


\section{DEVELOPMENT OF MULTICHANNEL hNIRS SYSTEM}

In an NIRS system, an optical probe (optode) is used to send near infrared light to the blood tissue and the reflected light is captured with another optode. The reflected light is significantly lower in intensity due to increased light absorption and scattering in the blood tissue [4]. As a result of this low intensity of light we have a poor signal-to-noise ratio (SNR). This difficulty can be overcome by using cooled CCD light detectors. Cooling allows for longer integration times without increasing the measurement noise. In my project I used the scientific-grade Ocean Optics QE 65000 spectrometer with the Hamamatsu TE-cooled 1044x64 element CCD array.

Light penetrates inside a spectrometer through a linear slit. The Fiber Optic Bundles direct the light from the tissue to the spectrometer through the slit. For an optimal light power (throughput) directed into the spectrometer the fiber bundle tip should be linearly shaped and aligned with the slit.

\subsection{Development of Fiber Optic Bundles}

The optic fibers that were developed for clinical fNIRS measurements are custom made from seven optic fibers. Each optic fiber is $0.50 \mathrm{NA}, \emptyset 400 \mu \mathrm{m}$ Core Multimode polymerclad fibers with broad UV/VIS/NIR spectral range of 400 to $2200 \mathrm{~nm}$ (Thorlabs, Newton, New Jersey) bundled together in a linear arrangement. These were made at lengths of $2 \mathrm{~m}$ and $6.5 \mathrm{~m}$ to accommodate the clinical procedures (TAVI experiments and fMRI respectively). This was needed as the commercial fibers available were of shorter lengths and lower throughputs. Figure 2.1 a. shows what the fiber bundles look like. 
The linear arrangement of the fibers (Figure 2.1 b.) was chosen to have an improved throughput from the spectrometers. The spectrometers (especially the QE 65000) have a linear slit to allow light into the inside of the spectrometer. The Fiber Optic Bundles direct the light from the source into the spectrometer through the slit. The fact that both the spectrometer opening slit and the Fiber Optic Bundles are linear ensures an optimal light power is directed into the spectrometer when the fiber bundle and the slit align. The numerical aperture (NA) of an optical system is a dimensionless number that characterizes the range of angles over which the system can accept or emit light. The

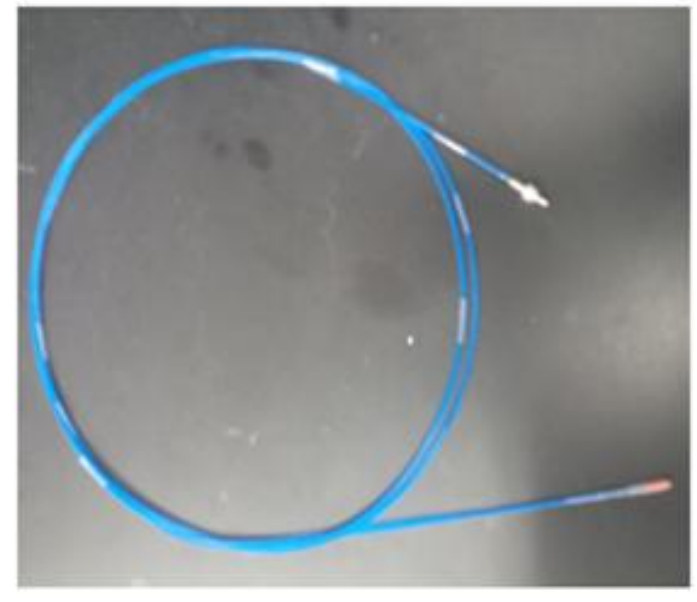

a. b.

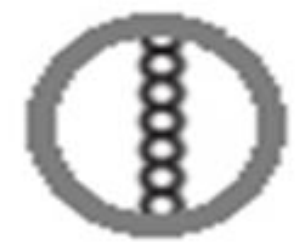

C.

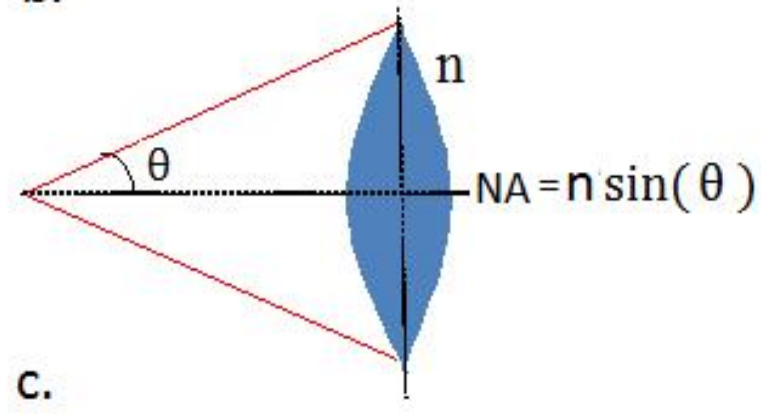

Figure 2.1 a. The optic fiber bundle and, b. The linear arrangement of the fibers, c. The numerical aperture (NA) of an optical system.

numerical aperture used for the fiber bundles $(\mathrm{NA}=0.5)$ indicates the high light gathering capacity of the fiber bundles as it is the highest range of angels within which light that is incident on the fiber will be transmitted along it. 


\subsubsection{Fiber Optic Bundle measurement}

All experimental probes used in our hNIRS measurements make use of fiber optic cables that were developed in our lab. The probes allow the NIRS measurements by guiding the incident and scattered light through these fibers. The custom made fibers were compared to the commercial fibers with respect to the spectrometer throughput and penetration with different neurocognitive tasks by testing on control subjects.

The power output from these fiber bundles was measured to be within the range of the maximum acceptable exposure by light $\left(\sim 200 \mathrm{~mW} / \mathrm{cm}^{2}\right)$. This ensures that all the experiments conducted using these optic fibers are safe and will not cause any harm to patients or subjects during experiments.

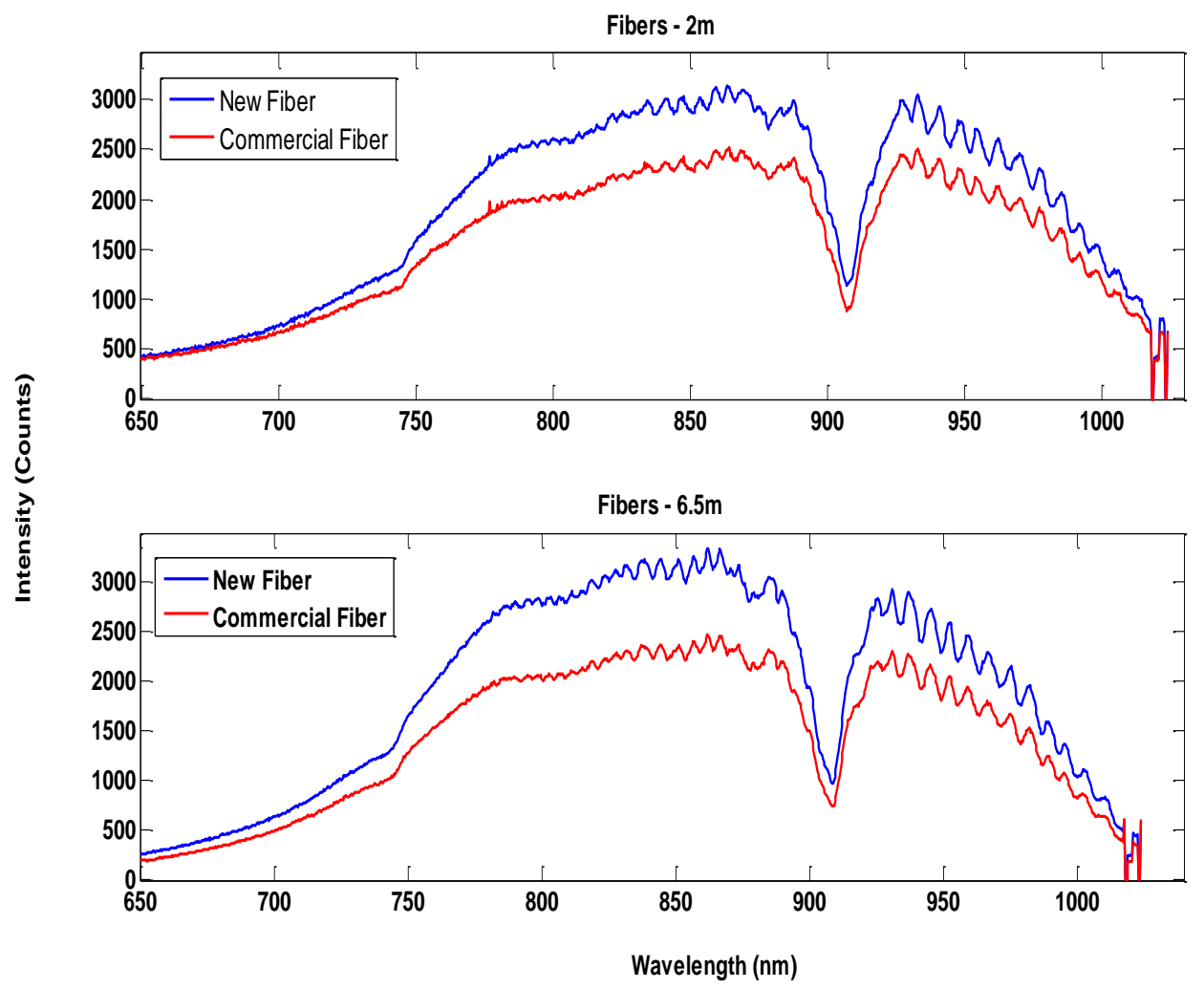

Figure 2.2 Comparison of fiber spectra. 
The results obtained using the commercial fibers (1m long) and custom-made fibers $(2 \mathrm{~m}$ and $6.5 \mathrm{~m}$ long) are shown in Figure 2.2. These figures show the spectra of the intensity versus wavelength for both custom-made and commercial fibers. From the figures it can be observed that the custom-made fibers (in blue color) have a significant increase in the spectrometer throughput. The two figures show a comparison of the custom-made fibers at different lengths ( $2 \mathrm{~m}$ and $6.5 \mathrm{~m}$ respectively) have each a better output than the commercial ones which were only available at shorter lengths. With these multiple length fibers we were able to develop optical fibers for hNIRS measurements that require adjustments with the MRI room and surgical environment during the TAVI measurements.

\subsection{Multichannel Setup and Measurements}

In this section we discuss the development of a multichannel hyperspectral NIRS (hNIRS) that is developed to overcome the inherent shortcomings that we have in multispectral NIRS (mNIRS). With hNIRS we can improve the quantitative accuracy of NIRS that is not met with mNIRS. hNIRS measurements reflect trends in changes of the tissue chromophore concentrations and blood oxygenation with an extended number of detectable chromophores. In this set up a near-infrared signal is gathered from the two sides of the brain (F7 and F8, Figure 3.3) using the multichannel measurements. The multichannel hNIRS set up includes a fiber optic switch (FOS), (FOS-2-Inline, Avantes, Louisville, CO), that connect the source to the two sides of the head enabling us the measurement of the two sides of the head simultaneously.

The source is a Fiber-Lite® DC950H Machine Vision Fiber Optic Illuminator (DolanJenner, Boxborough, Massachusetts). It operates at $100-200 \mathrm{VAC}$, is responsive to a 
frequency of $50 / 60 \mathrm{~Hz}$ and has a 150-watt quartz halogen, $21 \mathrm{~V}$ lamp. The lamp intensity is controlled by a rotary control. Two optic fibers, (Thorlabs, Newton, New Jersey), are used to connect the source to the FOS. The FOS is used to correct for light source drift and can be used automatically or via TTL pulse (Figure 2.4) from an external source. Another two bifurcated optic fibers (Thorlabs, Newton, New Jersey) are used to connect the FOS to the F7 and F8. Two spectrometers were connected to the F7 and F8 using optic fibers. The two spectrometers are two QE65000 scientific-grade spectrometers (Ocean Optics, Dunedin, Florida). (See Figure $2.3 \mathrm{a}$ and $\mathrm{b}$ below).

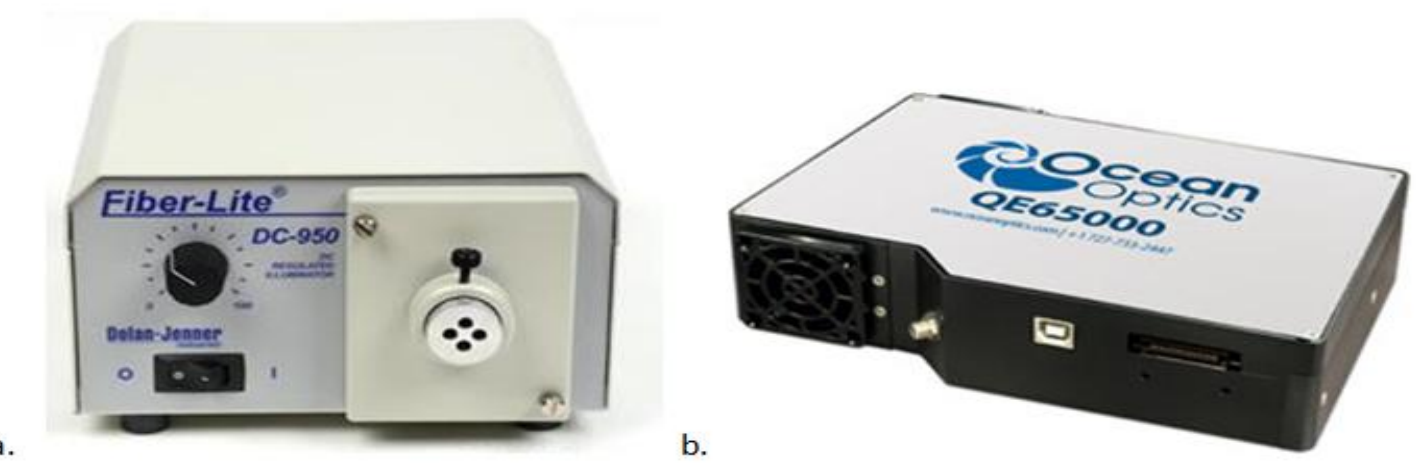

Figure 2.3 a. The Source (Fiber-Lite® DC950H Machine Vision Fiber Optic Illuminator) and, b. The QE65000 scientific-grade spectrometer (Ocean Optics, Dunedin, Florida)

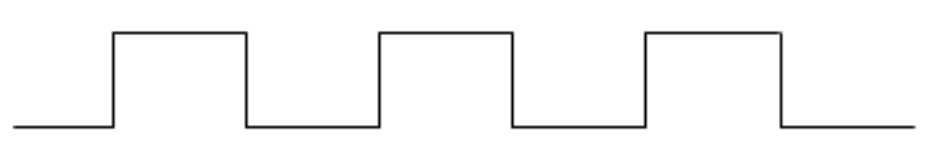

Figure 2.4 The Signal Diagram.

Since the spectrometers have several features Breakout Boxes were used to separate the spectrometer's 30-pin port easy access to all features of the spectrometer. The breakout boxes 


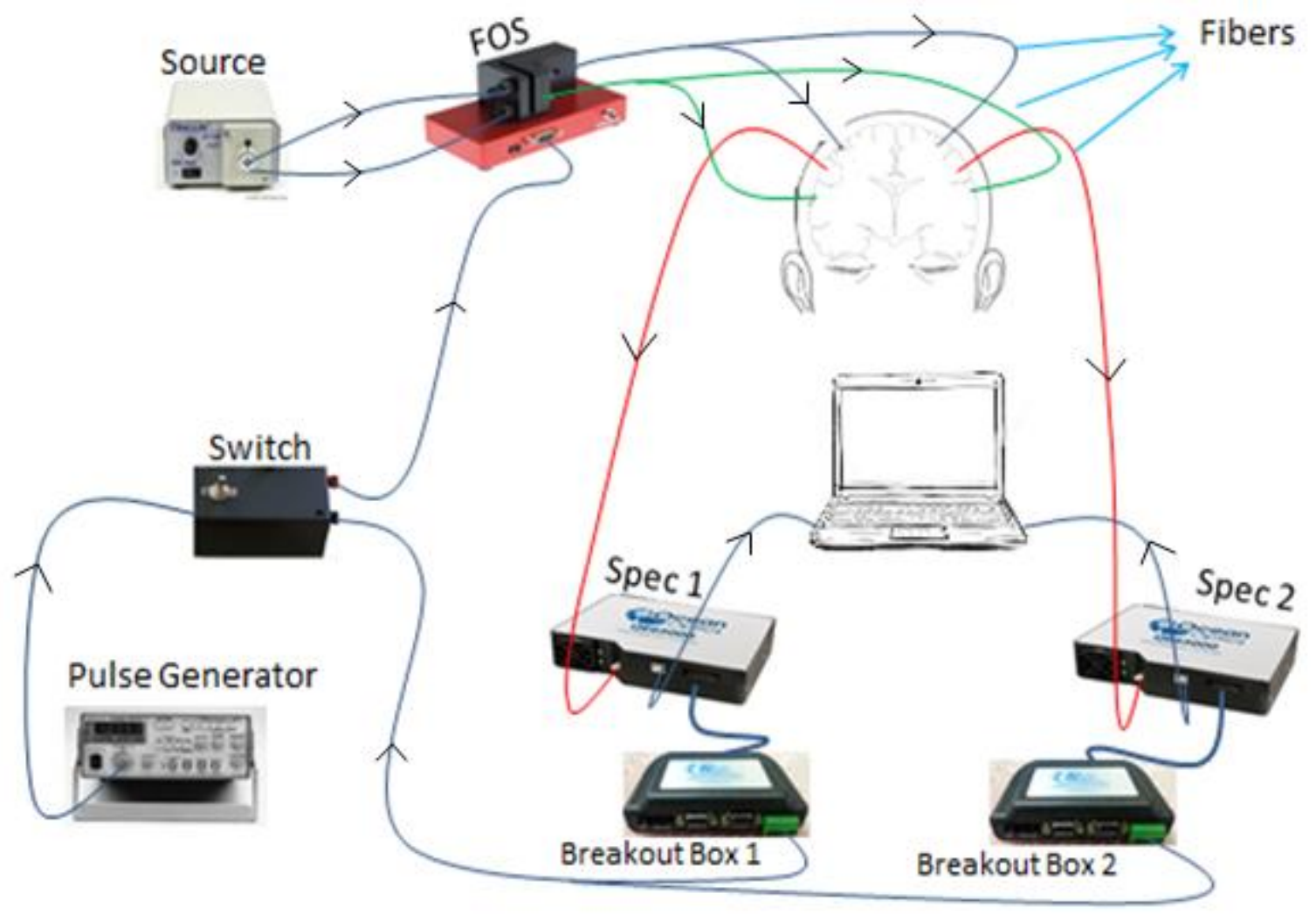

Figure 2.5 The Multi-Channel Experimental set up.

are HR4000 Break-Out Boxes (Ocean Optics, Dunedin, Florida). For external triggering, Pin 2 of the $\mathrm{J} 7$ connector of the Break-Out Box was used to separate the external triggering feature of the spectrometer to the triggering device. This allows synchronizing the data acquisition (scanning, acquisition, and transfer of data) with external triggering device. The triggering device in this experimental set up is a pulse generator, Synchronized Function Generator, model 288 (Wavetek, San Diago, CA). The pulse generator is a device that is used to generate pulses, especially rectangular pulses. They allow the control of the frequency, width and voltage level of the pulse and the delay with respect to an external trigger.

A switch was made to connect the pulse generator, the spectrometers via the breakout boxes and the FOS. This switch synchronizes these components of the experimental set up. 
With this set up we performed hNIRS measurements of the brain response to cognitive tasks, Eye Open/Close and Trail Making. These measurements were done on 11 control subjects.

\subsubsection{Multichannel hNIRS Test}

The novel hNIRS hardware design (Figure 2.5.) along with the data processing algorithm was developed to improve the signal-to-noise ratio of the hNIRS system and allow for the multichannel acquisition. These multichannel tests were performed to allow hNIRS measurements for different cognitive tasks that can be used for concussion detection. The cognitive tasks that were used are Eyes Open/Close, and Trail Making.
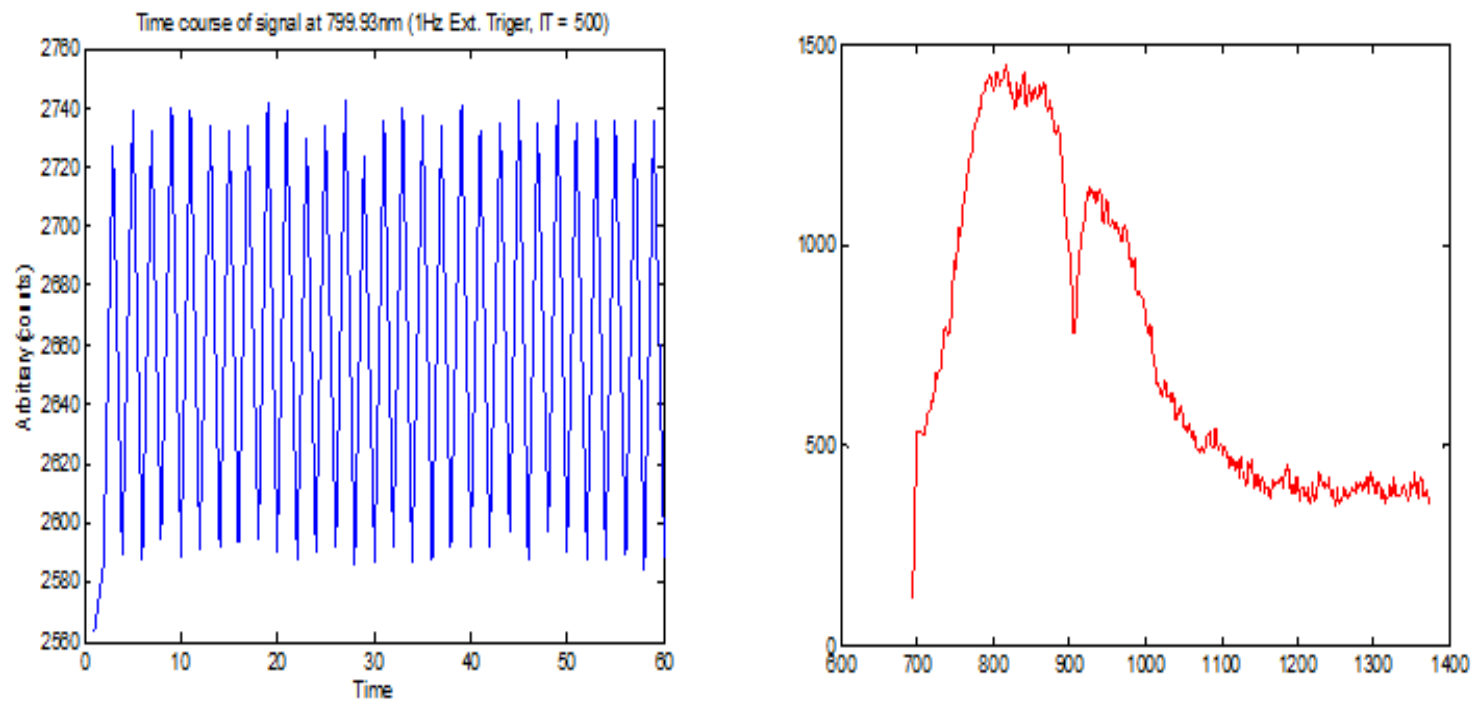

Figure 2.6 The Multichannel Raw data and b. Multichannel Raw Data Spectrum.

Figure 2.6. a. shows the raw data for the multichannel acquisition at $799.93 \mathrm{~nm}$ wavelength. This is a plot of the time course of the photon counts at the specific wavelength. And Figure 2.6. b. shows the raw spectrum for the multichannel acquisition at one specific time. This is the plot of intensity versus wavelengths. It can be observed from the raw 


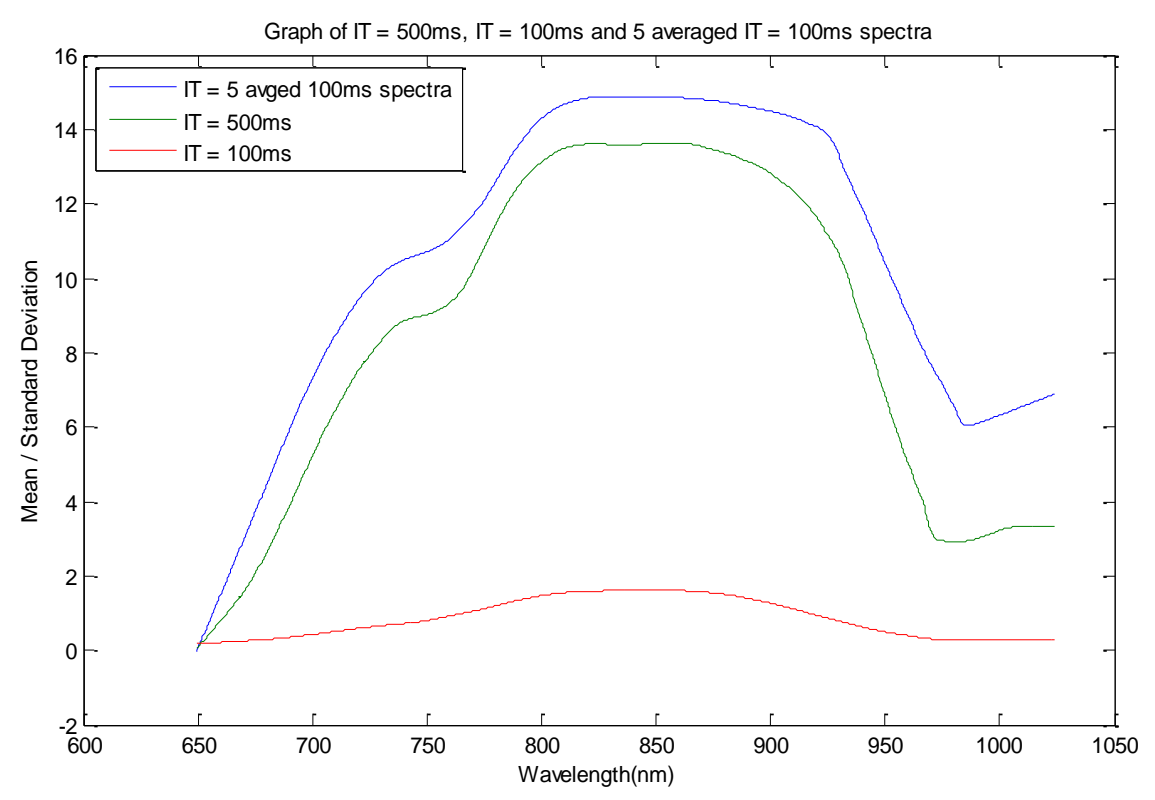

Figure 2.7 Multichannel SNR for IT at 500ms, 100s and average of five 100ms spectra. spectrum that the signal for the different chromophores are seen; the dip in the signal at $\sim 900 \mathrm{~nm}$ and $\sim 750 \mathrm{~nm}$.

As can be observed on Figure 2.7., the signal-to-noise ratio (SNR) of the spectra obtained at integration times of $100 \mathrm{~ms}$ (red curve) and 500ms (green curve) and the average of five 100ms (blue curve) show a significant increase in the intensity of the spectra of the averaged spectra at integration time of $100 \mathrm{~ms}$. The blue curves show a significant increase in the acquisition time, i.e we have a faster acquisition. This is of acute importance during clinical measurements where the time window for acquisition that is required is limited. These results indicate the suitability of the multichannel design in several clinical applications.

\subsection{Data Processing Algorithm}

All the hNIRS data collected were processed by a special hyperspectral signal processing algorithm on MatLab (Mathworks, MA, version R2013b). This helps us in extracting 
important information contained in several hundred raw data that correspond to several wavelengths.

The raw data is contaminated with motion artifacts due to extracerebral interferences and instances linked with experimental procedures. Hence, it has to be cleaned first. To do this we smooth the data over spectrally and temporally. We de-trend the spectra and perform ICA and remove artifacts and then back transform the data. Independent Component Analysis (ICA) is a method of blind-sorting data by minimizing covariance between components.

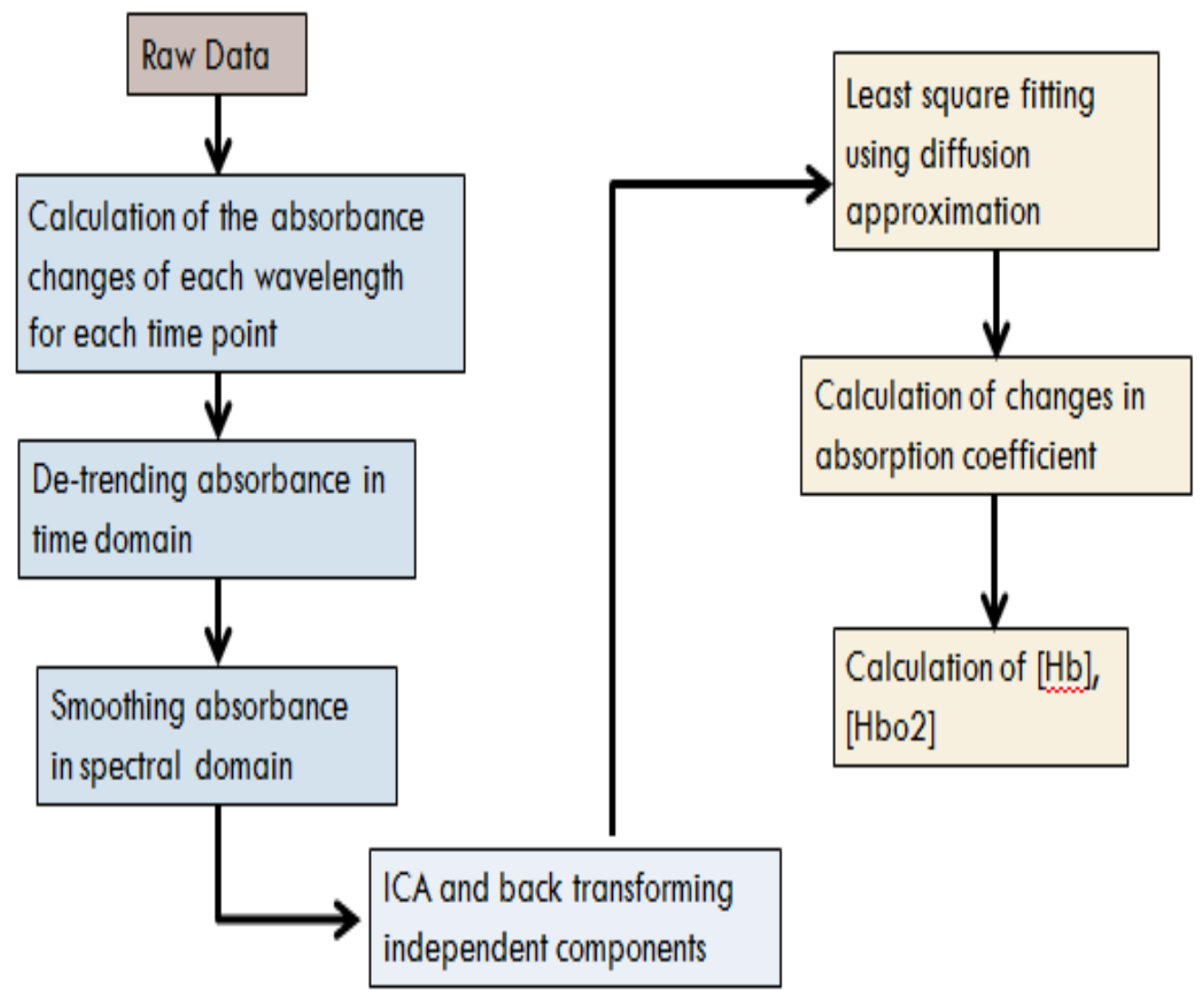

Figure 2.8 The data processing algorithm.

As the raw data contains hundreds of wavelengths and that a few of them contains most of the information, the use of ICA will remove most of the noise by keeping components that 
contain most of data (95\%). Once the raw data is cleaned, we calculate absorbance at each wavelength for each time point. Least squared fit using diffusion approximation and calculates change in absorption coefficients and extracts concentration of chromophores. 


\section{APPLICATION OF SYSTEM}

\subsection{Brain Activation Experimental Tasks}

The experimental tasks performed during the multichannel measurements, and the clinical applications (Occipital and fNIRS/fMRI measurements) were Eyes Open/Close and Trail Making. We had also the Clock Drawing task and Breath Holding during the fNIRS/fMRI measurement. The brain tissue oxygenation levels were investigated during these neurocognitive tasks.

The Eyes Open/Close (EOC) involves the simple procedure of alternating between opening the eyes and closing them when instructed. All measurements start with the eyes open for 30 seconds and are closed for the next 30 seconds. This is repeated 3 or 4 times. The neurocognitive task of Trail Making (TM) is sensitive to mental concentration and scaling difficulty, a test of visual search and cognitive flexibility, and includes three different levels of difficulty (Figure 3.1). The first one, the control task, is a simple joining of the numbers one and two four or five times. The next level is the joining of numbers from one to 25 and the most difficult level is the joining of numbers and the English alphabets, i.e. $1-\mathrm{A}-2-\mathrm{B}$ - and so on. The Clock drawing task, which is sensitive in screening for dementia, is the task of drawing an analog clock and baseline tracing. The subjects are tasked to make a drawing of the clock at a specific time. While the Breath Holding task is a task that is alternating between guided breathing, breath holding and then normal breathing. The breath hold is often taken for about 20 seconds. The purpose of using all these tasks is to observe that the hNIRS data is sensitive to these tasks. 

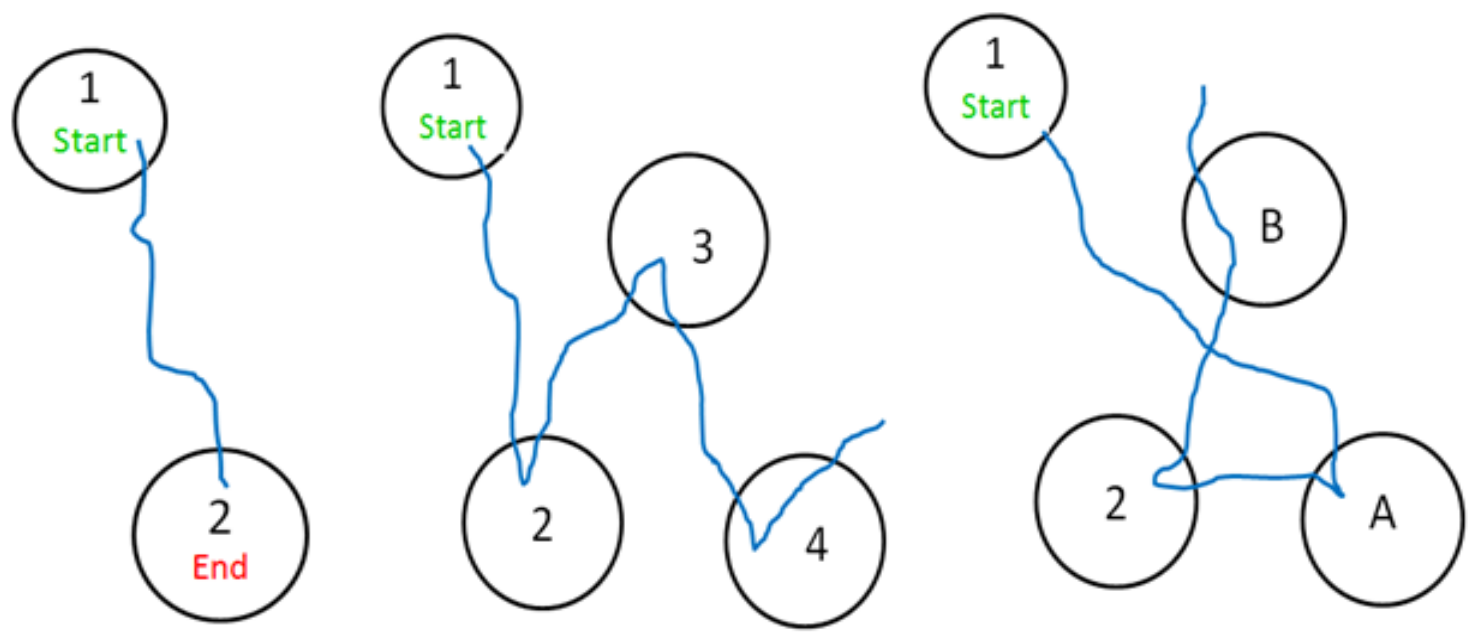

Figure 3.1 The neurocognitive task of Trail Making.

\subsection{Brain Response to Cognitive Tasks as measured by hNIRS}

In this part of the study we go through the results of the preliminary test done with the multichannel set up (Figure 2.5.). This set up included the above mentioned home-made Fiber Optic Bundles, which have the advantage of being long enough to fit in clinical setting such as the MRI room, fitted to probes that were connected to the two sides of the heads of the subjects. We performed several experiments to observe that hNIRS can measure cerebral response to stimuli that can be used for the concussion detection. The optodes were positioned according to the 10/20 system of positioning the EEG electrodes as shown in the figure below.

The experimental tasks that were performed were Eyes Open/Close and Trail Making on two channels (F7 and F8, Figure 3.3). The experimental set up, as shown in Figure 2.4., ensures the response to the stimuli is received from the two sides of the head (F7 and F8). The brain response to the cognitive task Eyes Open/Close is shown in Figure 3.4. This figure 
is the result of NIRS data processing of 11 subjects using algorithm (the fiDiff-Main algorithm) discussed in section 2.3.

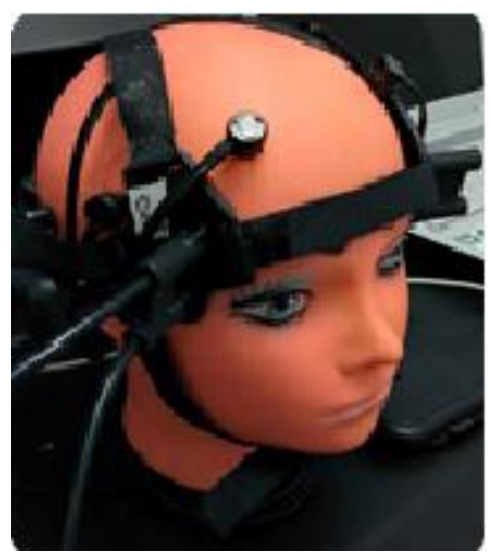

Figure 3.2 The Experimental headset showing the hNIRS and the EEG probes

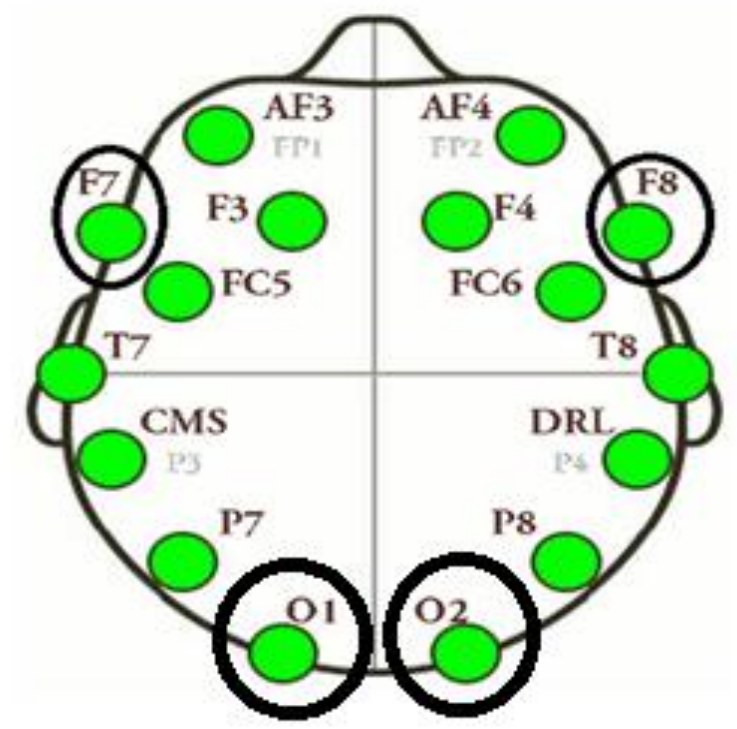

Figure 3.3 The electrode placement of the EEG (indicating F7 $(\mathrm{CH} 1)$ and F8 $(\mathrm{CH} 2)$ and Occipital regions $(\mathrm{O} 1$ and $\mathrm{O} 2)$ ).

The curves in these figures indicate the average of the all chromophores $(\mathrm{Hb}, \mathrm{HbO} 2$, $\mathrm{CCO}$ and $\mathrm{SO} 2$ ) of all subjects. The $\mathrm{x}$-axis represents the time of exposure in seconds and the $y$-axis indicates the concentration change relative to the baseline. 

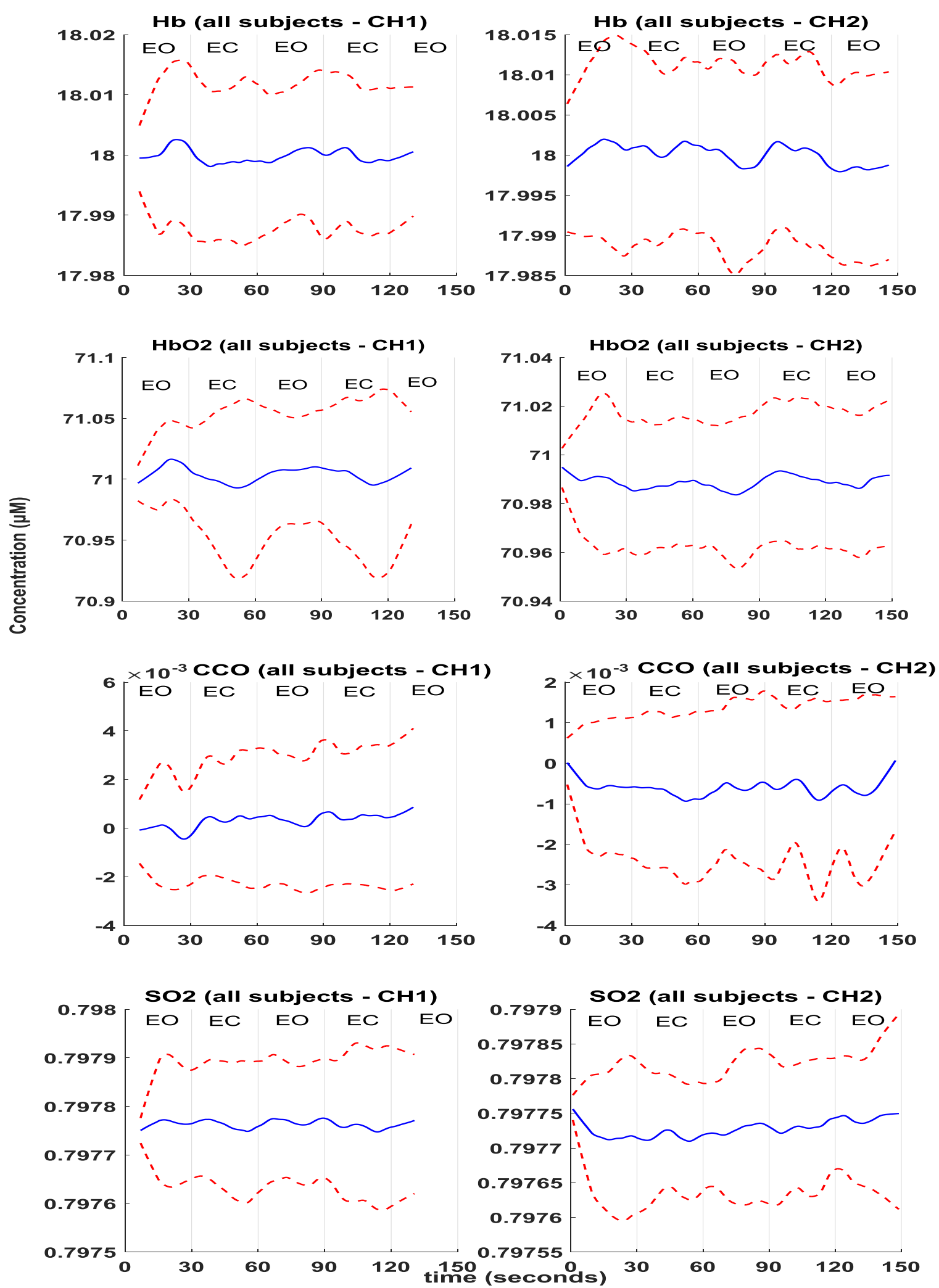

Figure 3.4 Averaged brain response to Eyes Open/Close task (Blue) and their corresponding standard deviation (Red) for a) Channel 1 and b) Channel 2. 
The lines shown in each graph at every 30 second interval show the change of the cognitive test from Eyes Open to Eyes Closed starting with Eyes Closed. This means that each graph is divided into regions where the subject is opening and closing their eyes alternatively with the first region being the Eyes Open region. The whole experiment was 150 seconds. The concentration for each chromophore averaged from all subjects, as shown in Figure 3.4, allows for an easier visual comparison between each of the chromophores from all subjects. By analyzing these data some observable trends can be seen in the figures.

Although the $\mathrm{Hb}$ and $\mathrm{HbO} 2$ plots in Figure 3.4. a. and b. are not as expected, there is a common similarity between the $\mathrm{Hb}$ for all subjects in channel 1 (F7) and that of channel 2 (F8). The same holds for the plots of $\mathrm{HbO} 2$ and $\mathrm{SO} 2$ plots in both channels. The CCO plots for all subjects in both channels did not show the expected trend of the cerebral blood oxygenation during Eyes Open/Close. The plots also indicate that the standard deviation of the data with respect to the signal changes to be large. But this was expected considering the small data set.

To test the significance of the hNIRS results, the eyes open regimes were separated from the eyes closed regimes for each individual subject and then they were subjected to a onesample t-test (ttest $(x)$ in Matlab), where $\mathrm{x}$ was the change in the chromophore concentration between a peak and the next dip or vice versa, against the eyes close regime to check whether they are significantly different. A 5 second gap at the end of each Eyes Open and Eyes Closed was taken, and the difference was calculated. The one-sample t-test was used to see whether the Eyes Open regime was different from the Eyes Closed regime in each transition and among the two transitions as well. Table 1 shows the results obtained for the t-test on all chromophores for both channels. 
Table 1. T-test result in micromolars $(\mu \mathrm{M})$, with p-values in brackets, for the comparison between the Eyes Open and Eyes Closed regimes of all subjects (EC1, EO1 - first Eyes Closed, first Eyes Open, and EC2, EO2 - second Eyes Closed, second Eyes Open).

\begin{tabular}{|c|c|c|c|c|}
\hline & Deoxy $(\mu \mathrm{M})$ & Oxy $(\mu \mathrm{M})$ & $\mathrm{CCO}(\mu \mathrm{M})$ & SO2 $(\mu \mathrm{M})$ \\
\hline \multicolumn{5}{|c|}{ Channel 1} \\
\hline EO1-EC1 & $0.0153(\mathrm{p}=0.047)$ & $0.0173(\mathrm{p}=0.192)$ & $-8.62 \mathrm{e}-4(\mathrm{p}=0.050)$ & $-8.35 e-6(p=0.757)$ \\
\hline EO2-EC2 & $-1.25 \mathrm{e}-4(\mathrm{p}=0.947)$ & $-0.0187(\mathrm{p}=0.132)$ & $7.71 \mathrm{e}-5(\mathrm{p}=0.802)$ & $-4.15 e-5(p=0.122)$ \\
\hline EO1-EC2 & $0.0041(p=0.114)$ & $0.0107(\mathrm{p}=0.479)$ & $-6.58 \mathrm{e}-4(\mathrm{p}=0.225)$ & $-1.21 \mathrm{e}-5(\mathrm{p}=0.800)$ \\
\hline EC1-EO2 & $-0.0011(p=0.578)$ & $0.0121(\mathrm{p}=0.307)$ & $1.27 \mathrm{e}-4(\mathrm{p}=0.613)$ & $3.78 \mathrm{e}-5(\mathrm{p}=0.246)$ \\
\hline \multicolumn{5}{|c|}{ Channel 2} \\
\hline EO1-EC1 & $0.0016(\mathrm{p}=0.296)$ & $-9.42 \mathrm{e}-4(\mathrm{p}=0.827)$ & $1.98 \mathrm{e}-4(\mathrm{p}=0.473)$ & $-7.45 e-6(p=0.397)$ \\
\hline EO2-EC2 & $3.96 \mathrm{e}-4(\mathrm{p}=0.766)$ & $-0.0035(\mathrm{p}=0.249)$ & $1.39 \mathrm{e}-4(\mathrm{p}=0.489)$ & $9.25 \mathrm{e}-6(\mathrm{p}=0.373)$ \\
\hline EO1-EC2 & $0.0033(\mathrm{p}=0.142)$ & $-0.0012(\mathrm{p}=0.783)$ & $2.37 \mathrm{e}-4(\mathrm{p}=0.496)$ & $-8.39 \mathrm{e}-6(\mathrm{p}=0.439)$ \\
\hline EC1-EO2 & $0.0013(\mathrm{p}=0.425)$ & $0.0032(\mathrm{p}=0.032)$ & $-9.92 \mathrm{e}-5(\mathrm{p}=0.742)$ & $-1.02 \mathrm{e}-5(\mathrm{p}=0.352)$ \\
\hline
\end{tabular}

The results of the one-sample t-test, as shown in Table 1, indicate that the hemodynamic response between eyes open and eyes closed are not statistically different with a significance level of $\mathrm{p}>0.05$ except for $\mathrm{Hb}$ during transition 1 (EO1-EC1) and $\mathrm{HbO} 2$ between transition 1 and transition $2(\mathrm{EC} 1-\mathrm{EO} 2)($ Deoxy $(\mathrm{p}=0.0047)$, Oxy $(\mathrm{p}=0.0032))$. The response from all chromophores for both channels and transitions are not significantly different which could be attributed to the small number of subjects that the experiment was tested on.

The brain response to the Trail Making task is shown in Figure 3.5. below. The plots indicate the time course of Oxy $(\mathrm{HbO} 2)$, Deoxy $(\mathrm{Hb})$, Cyt and $\mathrm{SO} 2$ amplitudes for both 

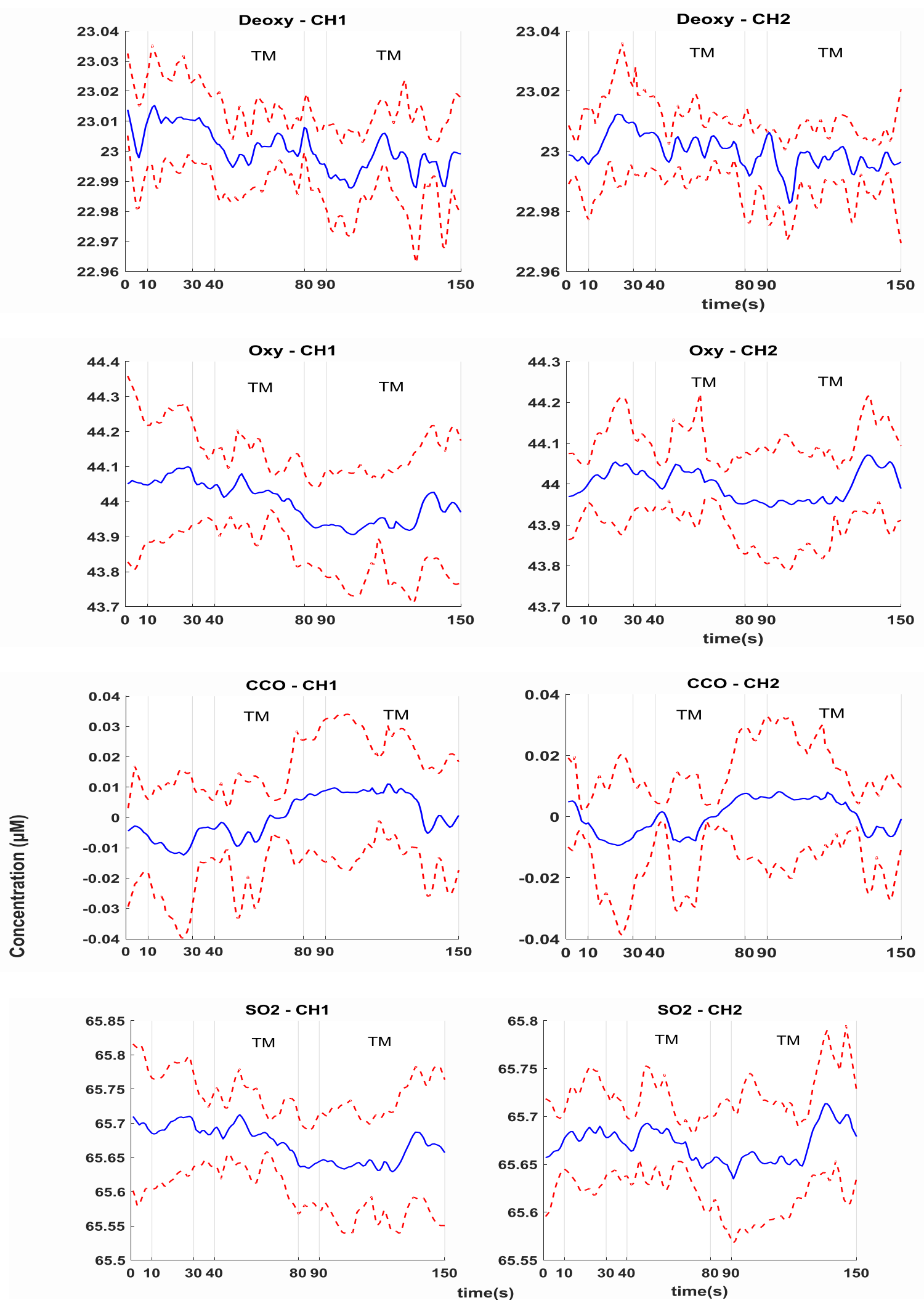

Figure 3.5 Averaged brain response (Oxy, Deoxy and Total) to Trail Making task (Blue) and their corresponding standard deviation (Red) for a) Channel 1 and b) Channel 2. 
channels 1 and 2. In each plot is indicated the trail making schemes of 20 seconds of control, 40 seconds of intermediate level (Trail A) and 60 seconds of difficult level (Trail B) of trail making. Each level is separated by a 10 seconds rest gap.

The plots show a large standard deviation which means that there is a large spread of values in the data than change in signal. And, though the expected results were not observed in all the results of the experiment, the plots show an increase in the Oxy during the difficult

phase of the Trail Making task (Trail B) for both channels while the Deoxy was decreasing. There is also repeatability between the two channels.

Table 2. T-test result (in $\mu \mathrm{M}$ ) of the average difference between Rest period and Trails $\mathrm{A}$ and $\mathrm{B}$, and between Trails A and B ( $\mathrm{p}$-values are indicated in brackets with $\mathrm{p}<0.05$ highlighted).

\begin{tabular}{|c|c|c|c|c|}
\hline & Deoxy $(\mu \mathrm{M})$ & Oxy $(\mu M)$ & $\operatorname{CCO}(\mu \mathrm{M})$ & SO2 $(\mu \mathrm{M})$ \\
\hline \multicolumn{5}{|c|}{ Channel 1} \\
\hline $\begin{array}{c}\text { Rest Before- } \\
\text { Trail A }\end{array}$ & $0.0022(\mathrm{p}=0.653)$ & $0.019(\mathrm{p}=0.124)$ & $-0.010(\mathrm{p}=0.241)$ & $0.039(\mathrm{p}=0.132)$ \\
\hline $\begin{array}{l}\text { Rest Before- } \\
\text { Trail B }\end{array}$ & $-0.0038(\mathrm{p}=0.363)$ & $-0.0605(\mathrm{p}=0.604)$ & $0.0098(\mathrm{p}=0.518)$ & $-0.027(\mathrm{p}=0.646)$ \\
\hline $\begin{array}{c}\text { Trail A- } \\
\text { Rest After }\end{array}$ & $-0.0077(\mathrm{p}=0.043)$ & $-0.0236(\mathrm{p}=0.452)$ & $0.0022(\mathrm{p}=0.327)$ & $-0.005(\mathrm{p}=0.786)$ \\
\hline \multicolumn{5}{|c|}{ Channel 2} \\
\hline $\begin{array}{c}\text { Rest Before- } \\
\text { Trail A }\end{array}$ & $0.0071(\mathrm{p}=0.294)$ & $0.0507(\mathrm{p}=0.356)$ & $-0.008(\mathrm{p}=0.414)$ & $0.019(\mathrm{p}=0.436)$ \\
\hline $\begin{array}{c}\text { Rest Before- } \\
\text { Trail B }\end{array}$ & $1.88 \mathrm{e}-4(\mathrm{p}=0.972)$ & $-0.084(\mathrm{p}=0.395)$ & $0.0111(\mathrm{p}=0.425)$ & $-0.043(\mathrm{p}=0.403)$ \\
\hline $\begin{array}{c}\text { Trail A- } \\
\text { Rest After }\end{array}$ & $0.0034(\mathrm{p}=0.451)$ & $-0.004(\mathrm{p}=0.734)$ & $1.1 \mathrm{e}-4(\mathrm{p}=0.940)$ & $-0.005(\mathrm{p}=0.572)$ \\
\hline
\end{tabular}

The average value of the NIRS data for each chromophore was used to test whether there was a difference between each chromophore changes in the regions when the subject was at 
rest against when the subject was engaged in the task. A gap of around 5 seconds was taken from the start of each region and also the end after Trails A and B and a one-sample t-test (ttest ( $x$ ) from Matlab), where $\mathrm{x}$ was the change in the chromophore concentration between a peak and the next dip or vice versa, was performed over the two channels between the rest period and each trail.

From Table 2 above, in terms of p-value, no significance difference between the two regions of rest period and trail A and B were observed for all of the chromophores except for $\mathrm{HHb}$ in channel 1 (Trail A - Rest after Trail A, $\mathrm{p}=0.043$ ). Although the change in concentration of Oxy and Deoxy for the Rest before Trail A and Trail A, for both channels, was not as expected (both changes show a decrease), the expected result is indicated for the most difficult part of the task (rest before Trail B and Trail B) for channel 2 and between intermediate and difficult tasks (Trail A vs Trail B) for channel 2. That is, we observe an increase in Oxyhemoglobin concentration while the Deoxyhemoglobin concentration is decreasing.

\subsection{Occipital Measurements}

The occipital measurement was performed using the set up shown below (Figure 3.6.). In this set up the probe (Figure 3.7. a) includes optic fibers (Thorlabs, Newton, New Jersey) arranged in an arc connected to a source (Fiber-Lite® DC950H Machine Vision Fiber Optic Illuminator) and a custom made optic fiber bundle (Figure 3.7. b) that is connected to a QE65000 scientific-grade spectrometer (Ocean Optics, Dunedin, Florida). The custom made fiber bundle is made of seven $0.50 \mathrm{NA}, \varnothing 400 \mu \mathrm{m}$ Core Multimode polymer-clad fibers with 
broad UV/VIS/NIR spectral range of 400 to $2200 \mathrm{~nm}$ (Thorlabs, Newton, New Jersey). These bundles were made at a length of $2 \mathrm{~m}$.

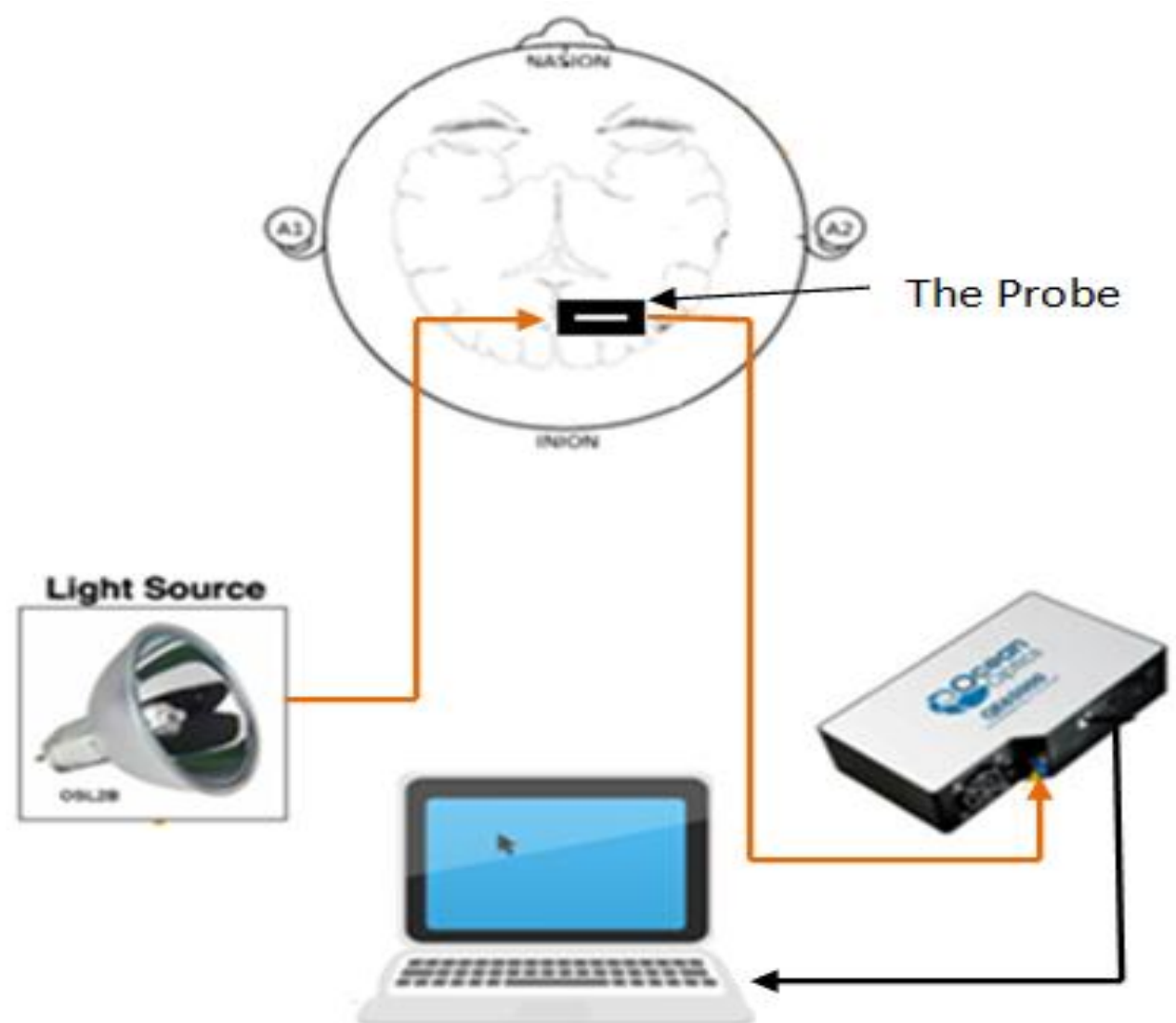

Figure 3.6 The experimental set up for Occipital measurement

The occipital lobe is one of the major lobes of the cerebral cortex [35] that contain the visual cortex of the brain. This is found at the back of the head and is most often full of hair (as shown in Figure 3.3 - O1 and O2). To avoid the problem caused by the presence of hair, the fibers are made as Brush-like bundles as shown in Figure 3.7. b. 


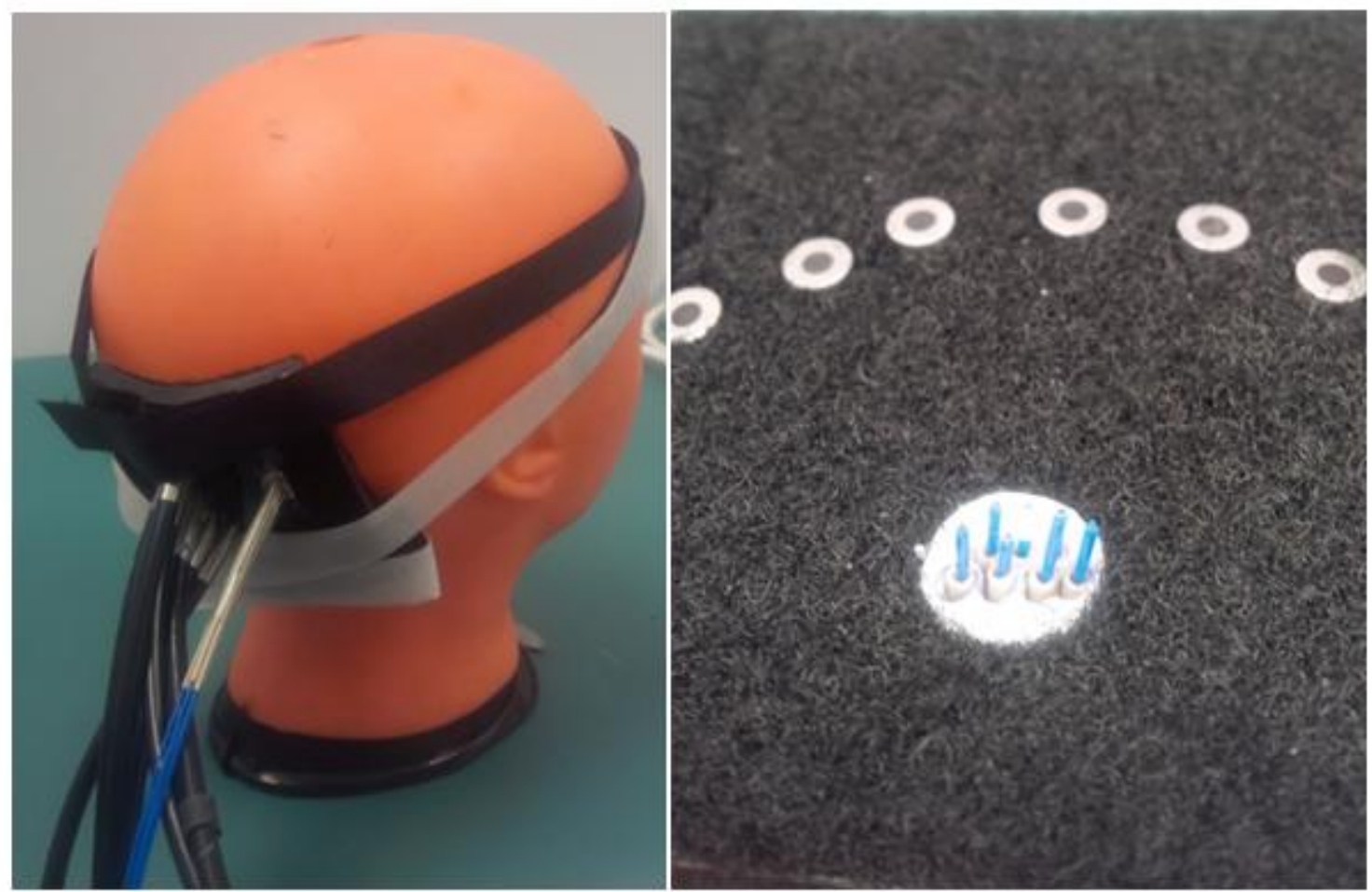

Figure 3.7 . a. Probe for Occipital measurement, and b. Optic fiber arrangement for the probe.

The hair structure is shown in Figure 3.8. The hair shaft goes deep into the skin and gets broader at the hair root which is located around the area where the blood vessels for the skin are. The arrangement and the size of the hair will be an obstacle for the transmission and receiving of light to and fro the blood vessels.

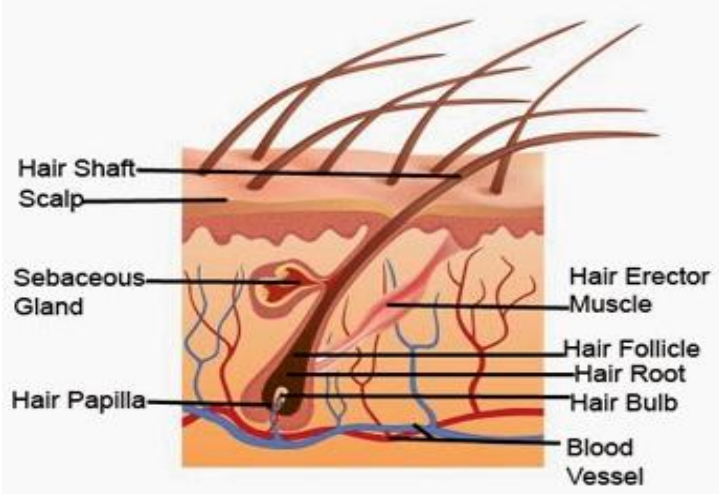

Figure 3.8 The structure of the Hair. 
The brush-like Fiber Optic Bundles that were made in our lab were used to measure the posterior lobe of the head. The challenges to be overcome during these experiments are that of the interference of the hair to the light. With our custom-made probes we were able to acquire useful data from subjects that have a distinct hair color.

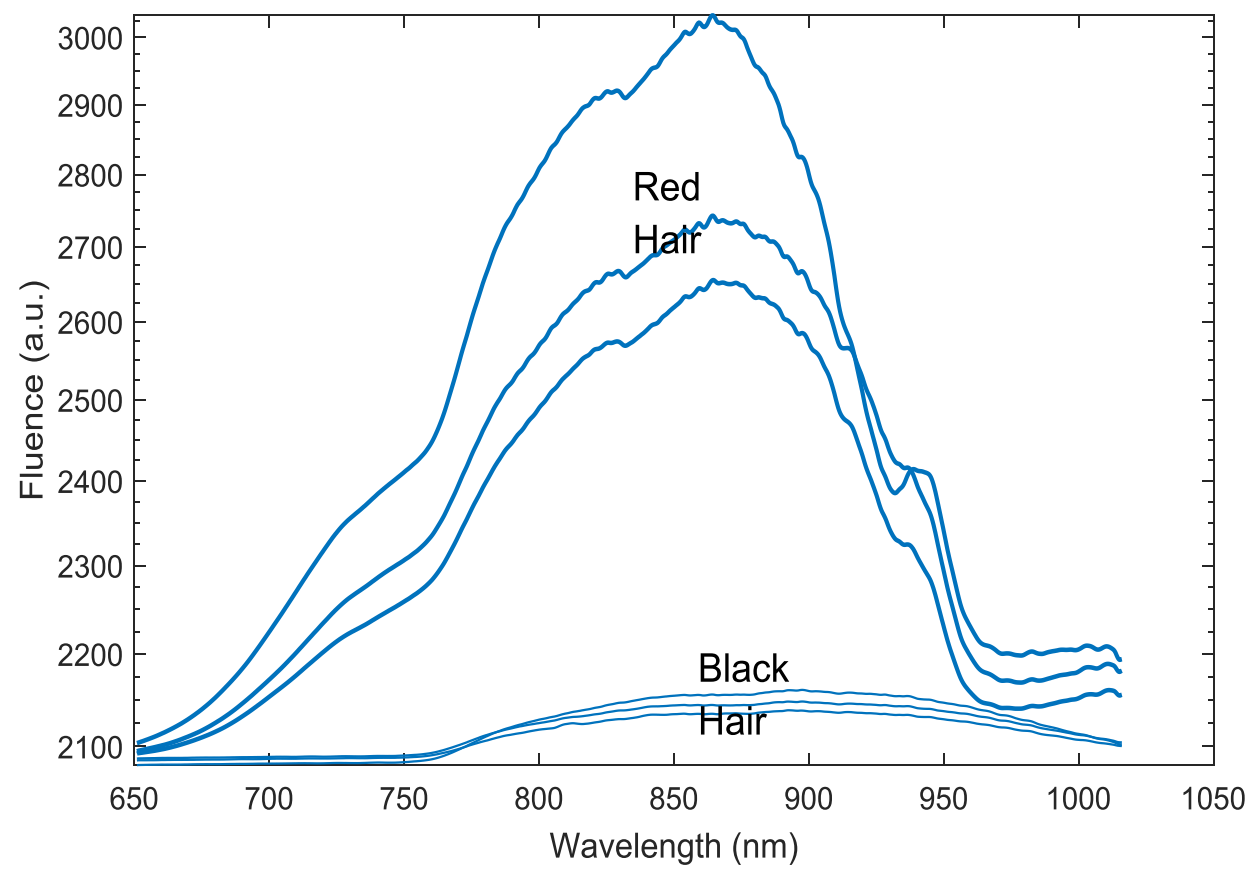

Figure 3.9 Spectra of the heads with Red and Black hair (integration time -1 second).

Although the fNIRS for subjects with black hair were not recognizable, for subjects with brown or lighter colors we had a very strong fNIRS signals. The figure above shows the significant difference between the spectra of red hair and black hair. All the distinctive features of chromophores in the NIR region are indicated in the red hair spectra while these features are absent for the black hair spectra. 

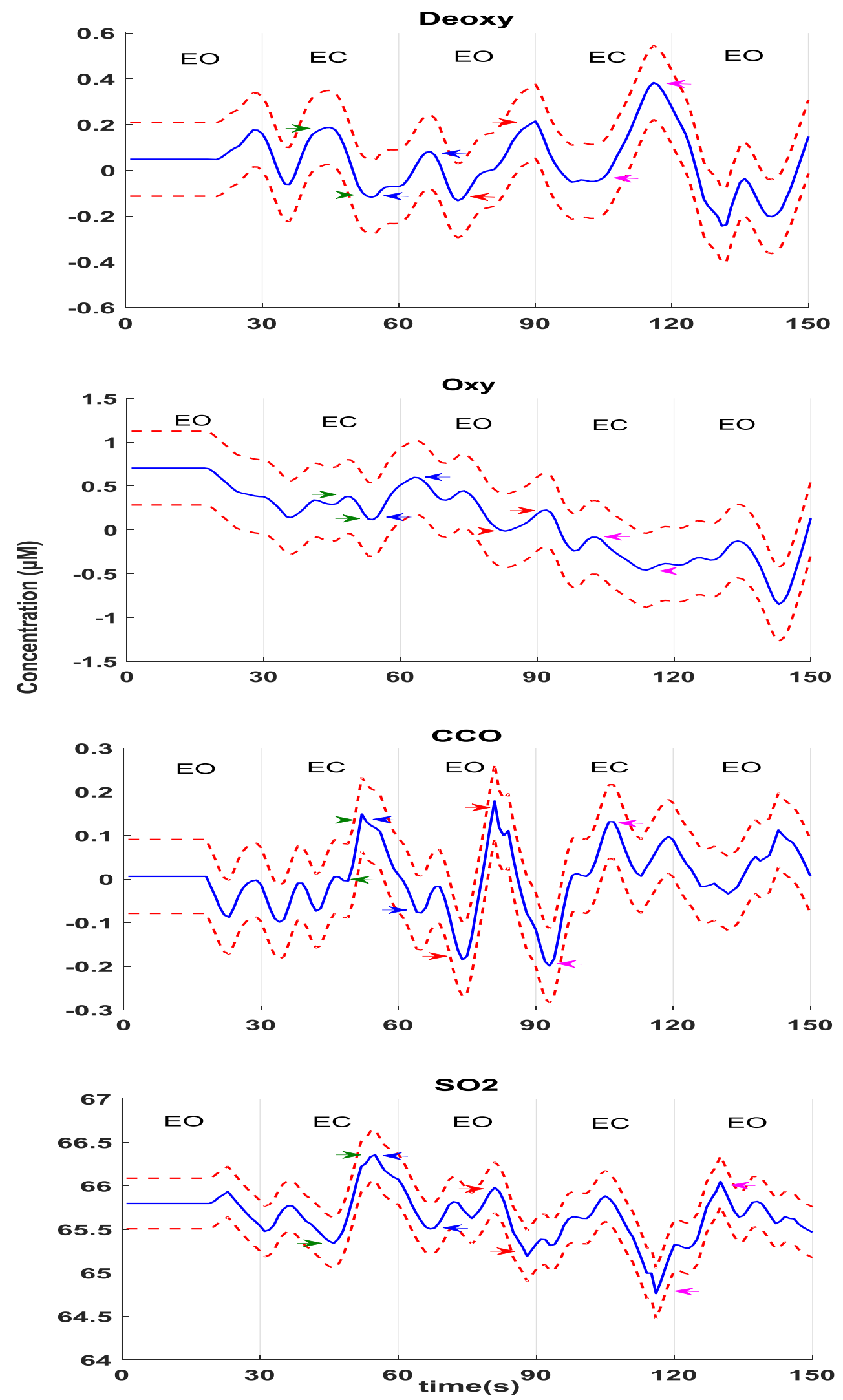

Figure 3.10 fNIRS plots of all chromophores during the occipital measurement. 
Figure 3.10. shows the brain response to Eyes Open/Close task that was performed for 150 seconds with a 30 second Eyes Open followed by a 30 second Eyes Close that was repeated three times. The plots show the time course of Deoxy ( $\mathrm{Hb})$, Oxy $(\mathrm{HbO} 2)$, Cytochrome C Oxidase, and SO2 smoothed at 5 points. The first 20s of all data were not used in the analysis (the flat curves at beginning of all plots) as the system would take about this time to adjust to the data acquisition and wouldn't reflect the changes due to the tasks. From the plots we can observe that Deoxy-Hemoglobin is a pattern of increasing in the mid of each Eyes Closed regime. And during the second Eyes Closed phase, the OxyHemoglobin and Deoxy-Hemoglobin are showing opposite trends as Oxy decreases Deoxy is increasing. The Cyt and SO2 plots at the bottom of Figure 3.10 show a much clearer response to the Eyes Open/Close task. We can observe an increase in the Cyt during the Eyes Close regime, with a much better increase in transition 2 .

Table 3. T-test results in $\mu \mathrm{M}$, with p-values in brackets, for the Occipital comparison between the Eyes Open and Eyes Closed regimes of all subjects (EC1, EO1 - first Eyes Closed, first Eyes Open, and EC2, EO2 - second Eyes Closed, second Eyes Open).

\begin{tabular}{|c|c|c|c|c|}
\hline & Deoxy $(\mu$ M) & Oxy $(\mu$ M) & CCO $(\mu$ M $)$ & SO2 $(\mu$ M $)$ \\
\hline EC1 & $-0.3199(\mathrm{p}=0.010)$ & $-0.2048(\mathrm{p}=0.2748)$ & $0.1863(\mathrm{p}=0.0374)$ & $0.9595(\mathrm{p}=0.0016)$ \\
\hline $\begin{array}{c}\text { EC1- } \\
\text { EO2 }\end{array}$ & $0.2260(\mathrm{p}=0.1413)$ & $0.3628(\mathrm{p}=0.2569)$ & $-0.2229(\mathrm{p}=0.0162)$ & $-0.8137(\mathrm{p}=0.0014)$ \\
\hline EO2 & $0.4275(\mathrm{p}=0.0179)$ & $0.2496(\mathrm{p}=0.1278)$ & $-0.2282(\mathrm{p}=0.0059)$ & $-0.6931(\mathrm{p}=0.0322)$ \\
\hline EC2 & $0.4441(\mathrm{p}=0.0242)$ & $-0.3846(\mathrm{p}=0.0726)$ & $0.0990(\mathrm{p}=0.0314)$ & $1.0424(\mathrm{p}=0.0103)$ \\
\hline
\end{tabular}

To reveal some Eyes Open/Closed task-triggered changes, the data were subjected to a one-sample t-test (ttest $(x)$ in Matlab), where $\mathrm{x}$ was the change in the chromophore concentration between a peak and the next dip or vice versa. Significance of changes 
between each deepest minimum and the next highest maximum or vise versa was calculated to check whether they are significantly different. A similar procedure, as was done for the multichannel measurement, was performed for the t-test here as well. The one-sample t-test on the difference between each minimum and the next maximum for the span of five time points was done. Table 3 shows the results obtained for the t-test on all chromophores to see whether the Eyes Open regime was different from the Eyes Closed regime in each phase and among the two phases as well. These are indicated by the arrows in the plots above (Figure 3.10.). The results of the t-test (Table 3) indicate that the hemodynamic response between eyes open and eyes closed are statistically different $(\mathrm{p}<0.05)$ for Deoxy-hemoglobin ( $\mathrm{HbO} 2)$, Cytochrome C Oxidase (CCO) and the Oxygen saturation (SO2). From the test it is revealed that Oxy-hemoglobin did not yield any statistically significant results $(\mathrm{p}>0.05)$.

\subsection{Measurements During Transcatheter Aortic Valve Implantation}

hNIRS measurement was done during TAVI (Transcatheter Aortic Valve Implantation) surgery. This measurement is a non-invasive method that is done simultaneously with the measurement of blood oxygen saturation (rSO2) using Nonin Equinox 7600. TAVI is a clinical procedure done on patients with severe aortic stenosis when the patients are unable to go through an open-heart surgery. During this procedure an aortic valve is implanted into the heart using a balloon catheter, a long narrow tube. This catheter is often inserted through the groin into a large blood vessel. Aortic stenosis is a condition where the aortic valve cannot open and close properly. This condition puts extra strain on your heart and can result in breathlessness, swollen ankles, chest pain, dizziness, and sometimes, blackouts. The valve is made of natural tissue from the heart of either a cow or a pig. The natural tissue is reengineered and attached to a flexible expanding mesh frame. In order to implant it into the 
heart, the valve is squeezed around or inside a catheter. The catheter is then inserted and guided to the aortic valve opening in your heart where it is implanted over your existing valve. Once the new valve is implanted, the catheter is removed. The new valve starts working right away [36].

During the procedure, the valve is positioned after extremely rapid transvenous ventricular pacing. This was momentarily induced in the patient for approximately 15-20 seconds. The purpose of the pacing was so that the heart was placed in a near immobile state so that the valve may be implanted. The diminished cardiac output leads to a halt of effective blood circulation to the rest of the body and brain, which closely resembles cardiac arrestlike conditions [36].

\subsubsection{Clinical Set Up}

fNIRS signal was collected from the TAVI patients using the experimental set up shown below (Figure 3.11.) . This set up is based on the two-layer model that uses two spectrometers (the primary and secondary spectrometers) to separate the information obtained from the brain and the skull.

The probe is placed on the forehead at the F7 or F8 positions. It consists of two detector optic fibers placed at a distance of $1 \mathrm{~cm}$ and $2.8 \mathrm{~cm}$ from two source optic fibers as shown in Figure 3.12. The detector fibers are made from seven $0.50 \mathrm{NA}, \varnothing 400 \mu \mathrm{m}$ Core Multimode polymer-clad fibers with broad UV/VIS/NIR spectral range of 400 to $2200 \mathrm{~nm}$ (Thorlabs, Newton, New Jersey). They are custom made so that they are each $2 \mathrm{~m}$ long and one end of each fiber is made to have a linear arrangement so that can be aligned with the slit of the spectrometer and give an increased throughput. 


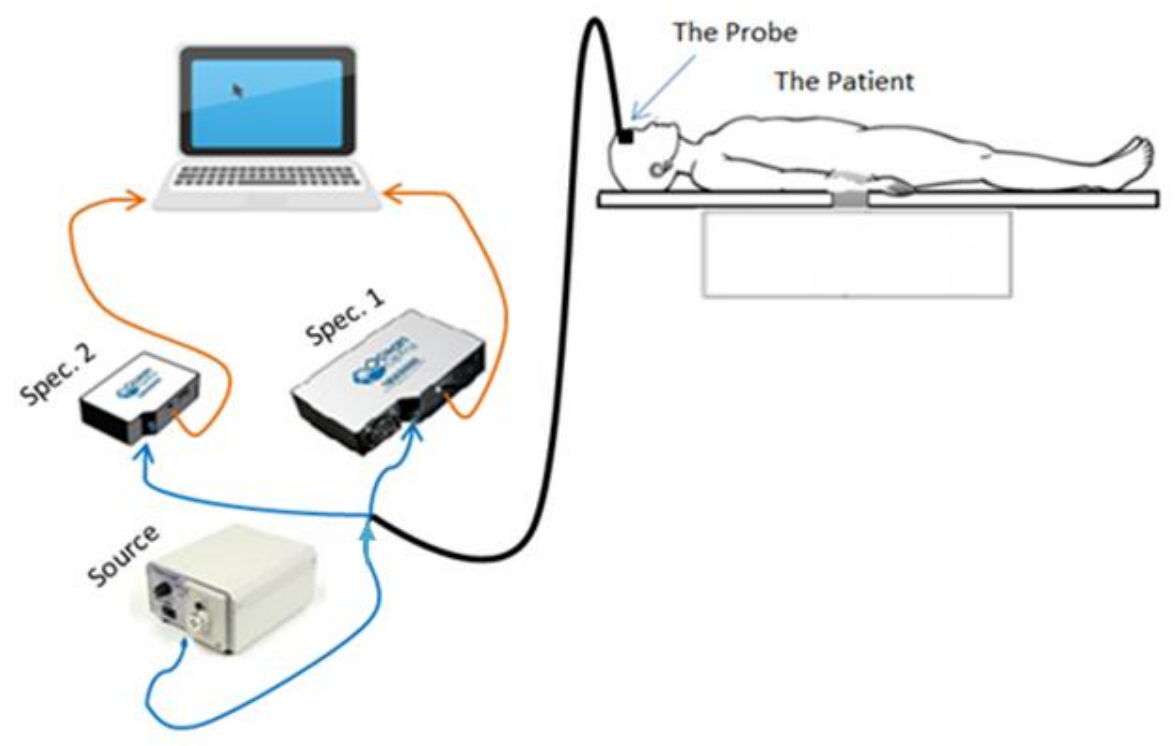

Figure 3.11 TAVI experimental set up.

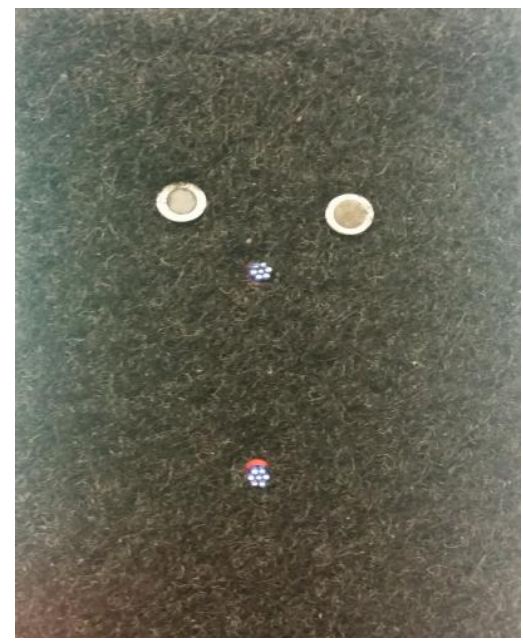

Figure 3.12 The TAVI measurement Probe.

One of the detector fibers that is at a distance of $1 \mathrm{~cm}$ from each source fiber is connected to the secondary spectrometer, the USB4000 Fiber Optic Spectrometer (Ocean Optics, Dunedin, Florida) and the other detector fiber that is at $2.8 \mathrm{~cm}$ from both source fibers is 
connected to the primary spectrometer, the QE65000 scientific-grade spectrometer (Ocean Optics, Dunedin, Florida). This spectrometer has high SNR with a spectral range between $200 \mathrm{~nm}$ and $1100 \mathrm{~nm}$. Both source fibers are the commercially available Straight Single Fiber Optic Light Guide (Dolan-Jenner, Boxborough, Massachusetts). These optic fibers are made of thousands of fine fibers that are tightly bundled together into a $5 \mathrm{~mm}$ diameter tube (Single Straight Flexible Light Guides Manual). We used the Fiber-Lite ${ }^{\circledR}$ DC950H Machine Vision Fiber Optic Illuminator as a source of light.

The hNIRS experiment was done in conjunction with mNIRS measurements performed by Cardiac researchers from St. Michael's Hospital (RESCUE). The mNIRS measurement monitors the cerebral oxygen blood saturation, the regional Oxygen Saturation (rSO2) of the patient by using a mNIRS Oximeter, the Nonin Equanox 7600 Regional Oximeter. A sensor (the Nonin Regional Oximetry Equanox Advance Adult/Pediatric 8004 CA sensor) is placed to the forehead of the patient and connected to the Nonin oximeter.

Clinical settings that were put into consideration during the measurement were that to allow the C-Scan to freely move over the patient. Our equipment has also to be set up in such a way not to interfere with the movements of the Anesthesiologists that were placed on the same side as the hNIRS and Nonin group and allow the surgeons to move the patient freely. The $2 \mathrm{~m}$ long optic fibers used were long enough to enables to give lesser restriction on movement and allow the transmission of the light from the source to the subject and back to the spectrometers. The entire set up was placed in a double shelf table which gives enough space to make an easy set up and start the procedure quickly in the small window of time given (see Figure 3.13.). 


\subsubsection{Clinical Procedures}

The main focus during the clinical procedures is to keep a safe fNIRS/mNIRS data acquisition that ensures a minimal interference with TAVI operation teams. As Figure 3.13. shows, the clinical environment involves the patient lying on the surgery bed and the fNIRS/mNIRS equipments on the head side of the bed along with the Anesthesiologist. This keeps the surgeon to freely move the C-Scan and operate on the patients. Our equipments

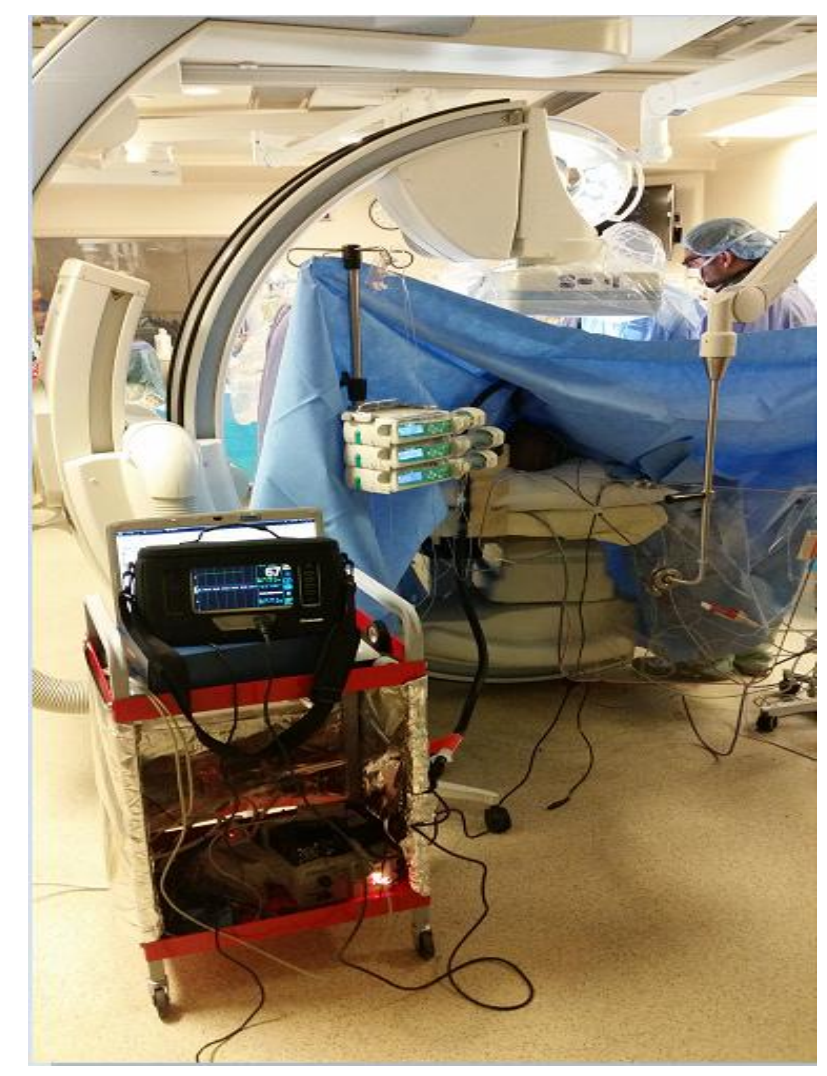

Figure 3.13 The TAVI experimental set up including the C-Scan.

would be ready while the Anesthesiologist is administering the necessary drugs to get the patient to be unconscious. Only when the Anesthesiologist makes sure that the patient is sleeping can we put our probes on the patient's head. To make an easy set up of the probe on 
the patients head, we made a two part head piece. One part goes to the bottom of the patient's head and the other part is put on the forehead (F7 or F8) and is attached to the bottom part without moving the patient.

When ready for data acquisition, first the mNIRS probe (Nonin sensor) is placed on one side of the patient's head. The fNIRS probe (Figure 3.12.) is then placed on the other side of the patient's head level with the Nonin sensor and its rubber strap will help to hold both probes in place during the surgery. A consent form to participate in the research study of evaluating the use of NIRS was obtained from 10 patients and the experiments went ahead. The software SpectraSuite (Ocean Optics, Dunedin, Florida) was used to collect hNIRS data from these TAVI patients. The data acquisition was started in tandem with the mNIRS data collection and the time range was put to be more than an hour as the TAVI surgery usually takes about 45 minutes.

\subsubsection{Clinical Results}

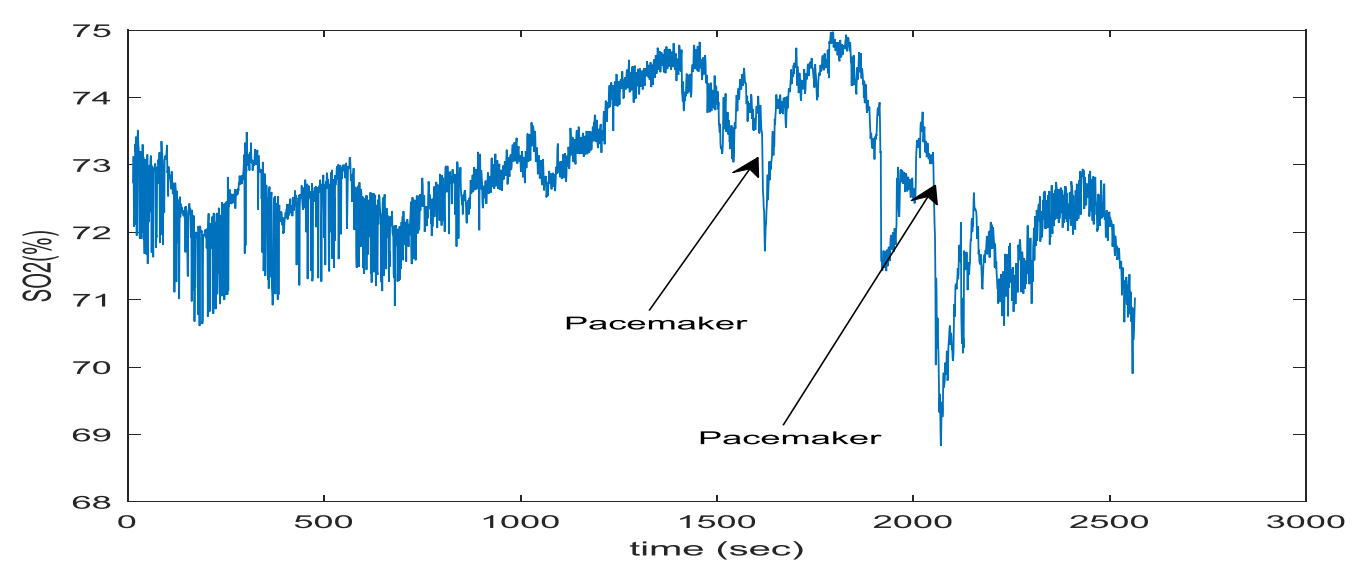

Figure 3.14 Measurement of the Cerebral Oxygen Saturation.

Figures 3.14. above and Figure 3.15. below show measurements of the cerebral oxygen saturation, $\mathrm{Hb}$, and $\mathrm{HbO} 2$ during cardiac surgery, TAVI. These are time course series of the 
$\mathrm{SO} 2$ concentration (percentage) and $\mathrm{Hb}$ and $\mathrm{HbO} 2(\mu \mathrm{M})$. Although the $\mathrm{Hb}$ and $\mathrm{HbO} 2$ curves are not the expected increase in $\mathrm{Hb}$ while $\mathrm{HbO} 2$ is decreasing or vise versa, the figures indicate a clear deep during the periods when the pacer is on. In the plot of the $\mathrm{SO} 2$, we had two pacer ons and these are measured as a significant drop in the graph. We had three significant drops in the graphs of the $\mathrm{Hb}$ and $\mathrm{HbO} 2$ plots indicating the three pacer ons.

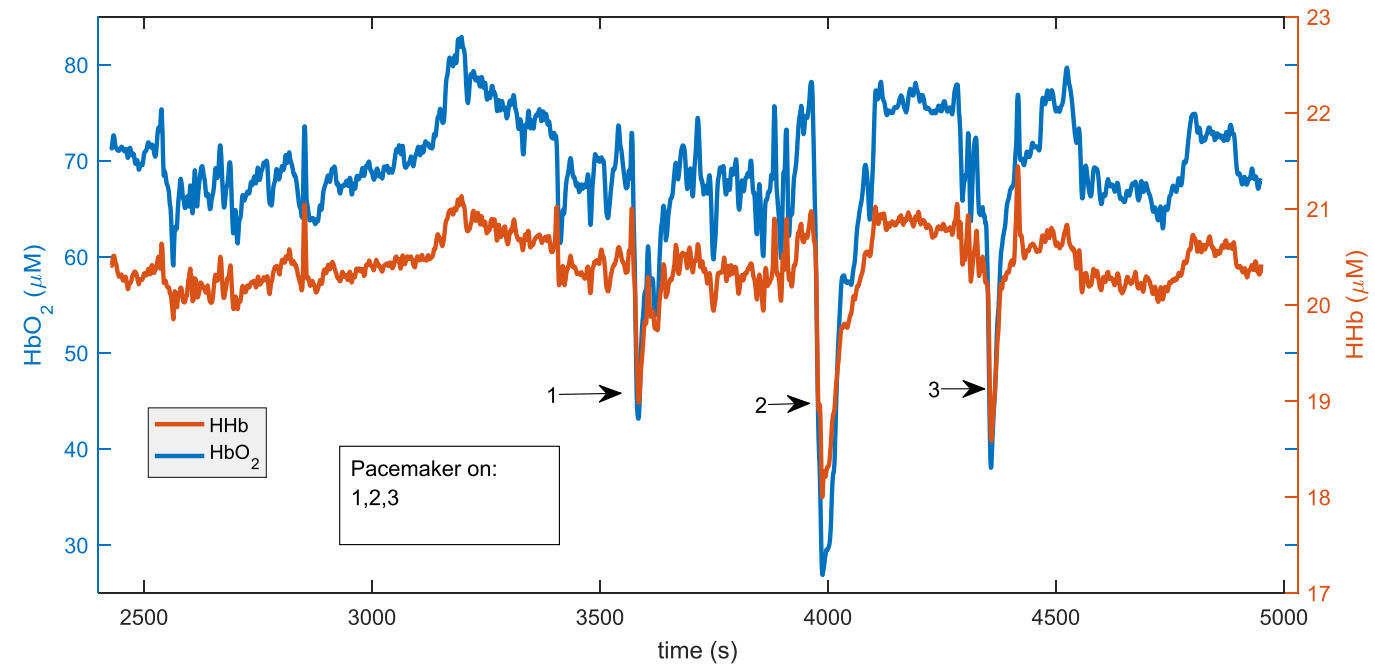

Figure 3.15 Measurement of cerebral $\mathrm{Hb}$ and $\mathrm{HbO} 2$ during TAVI.

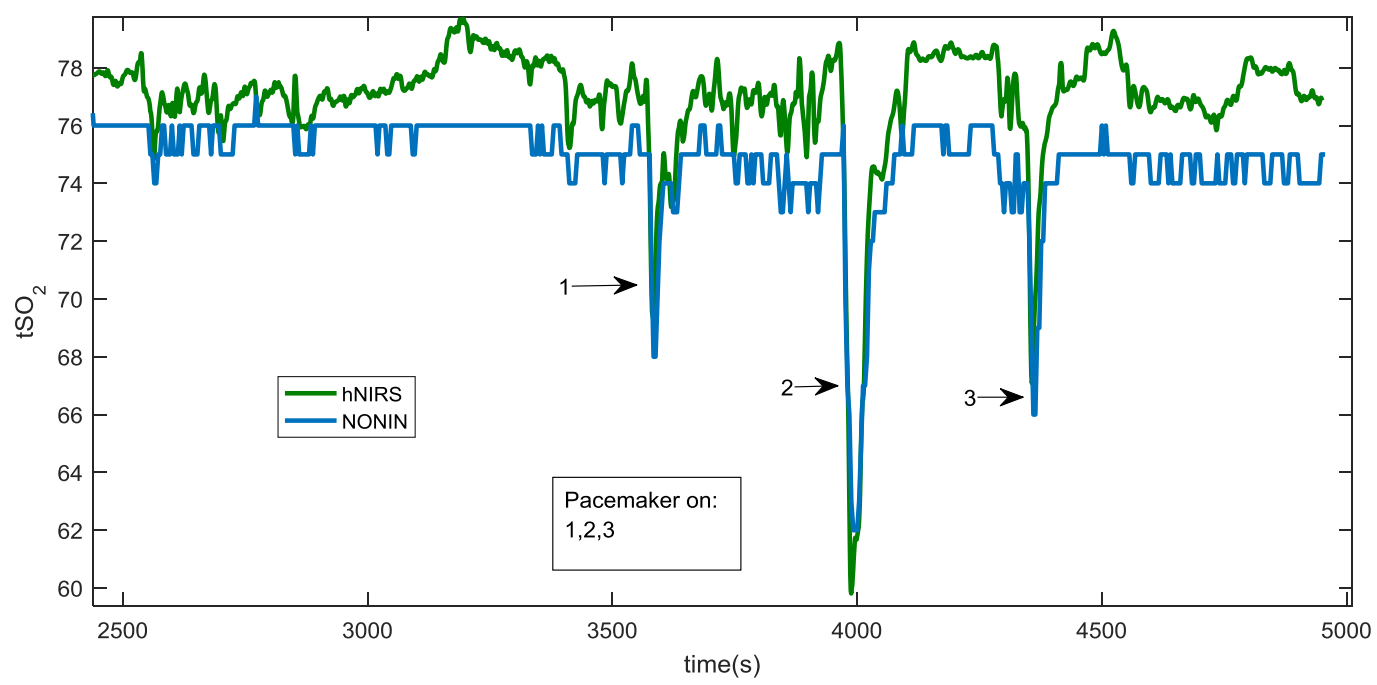

Figure 3.16 . hNIRS versus Nonin measurement. 
Figure 3.16. shows a plot of the hNIRS measurement plotted on top of that obtained from Nonin measurements. This is the time course plot of the oxygen saturation of the patient throughout the surgery. From this graph there can be observed that there is almost perfect consistency in the drop of the [tSO2] during the introduction of the pacer. This is seen similarly in the three occasions that the pacer is put on.

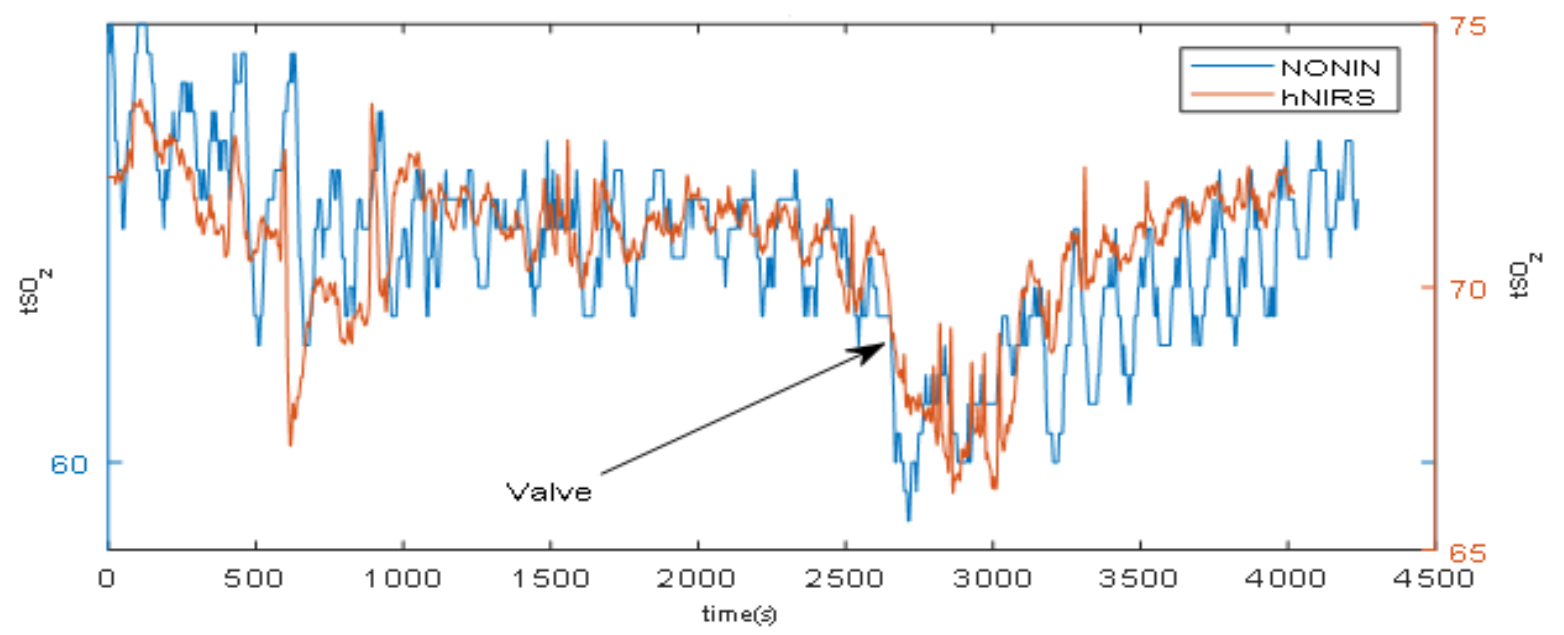

Figure 3.17 Patient 1: Comparison of SO2 measured by mNIRS and hNIRS.

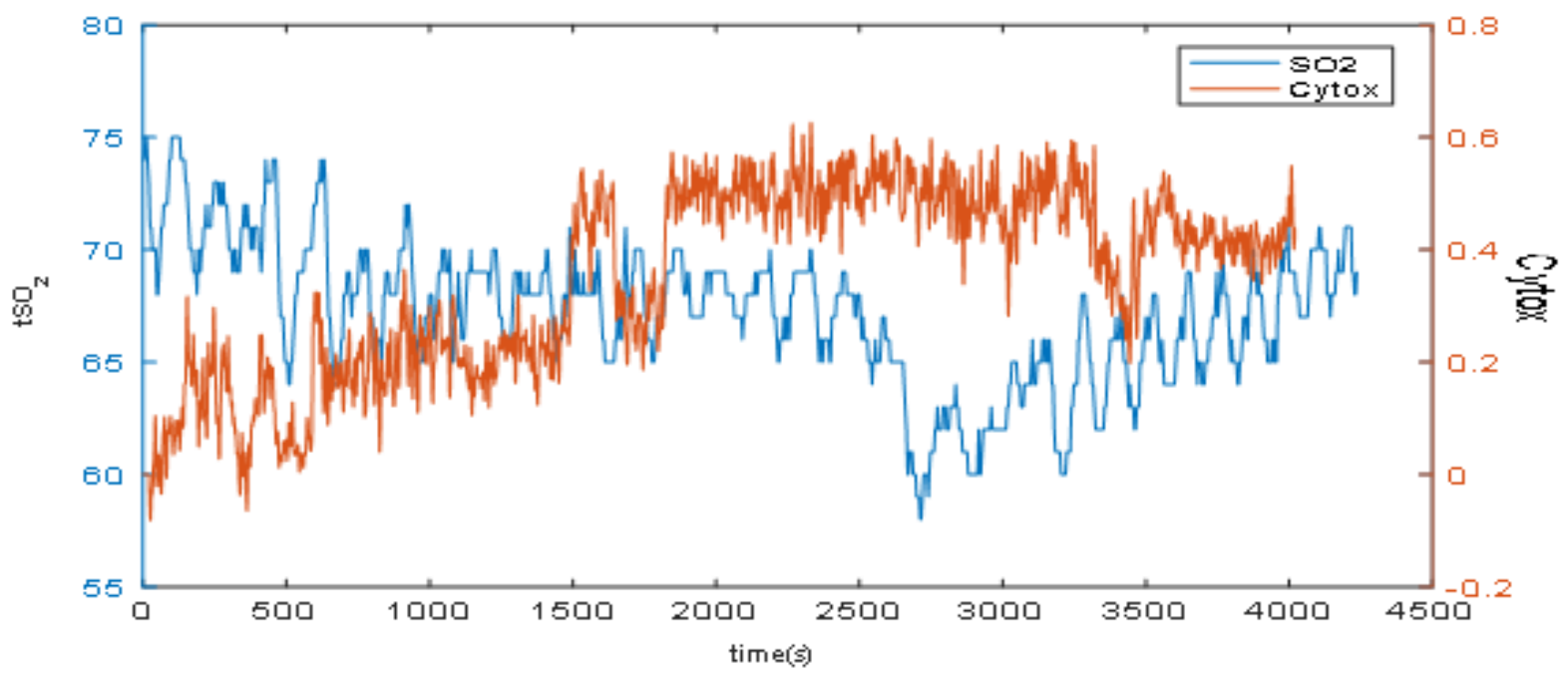

Figure 3.18 Patient 1: Comparison of SO2 measured by mNIRS and Cyt-Ox measured by hNIRS. 


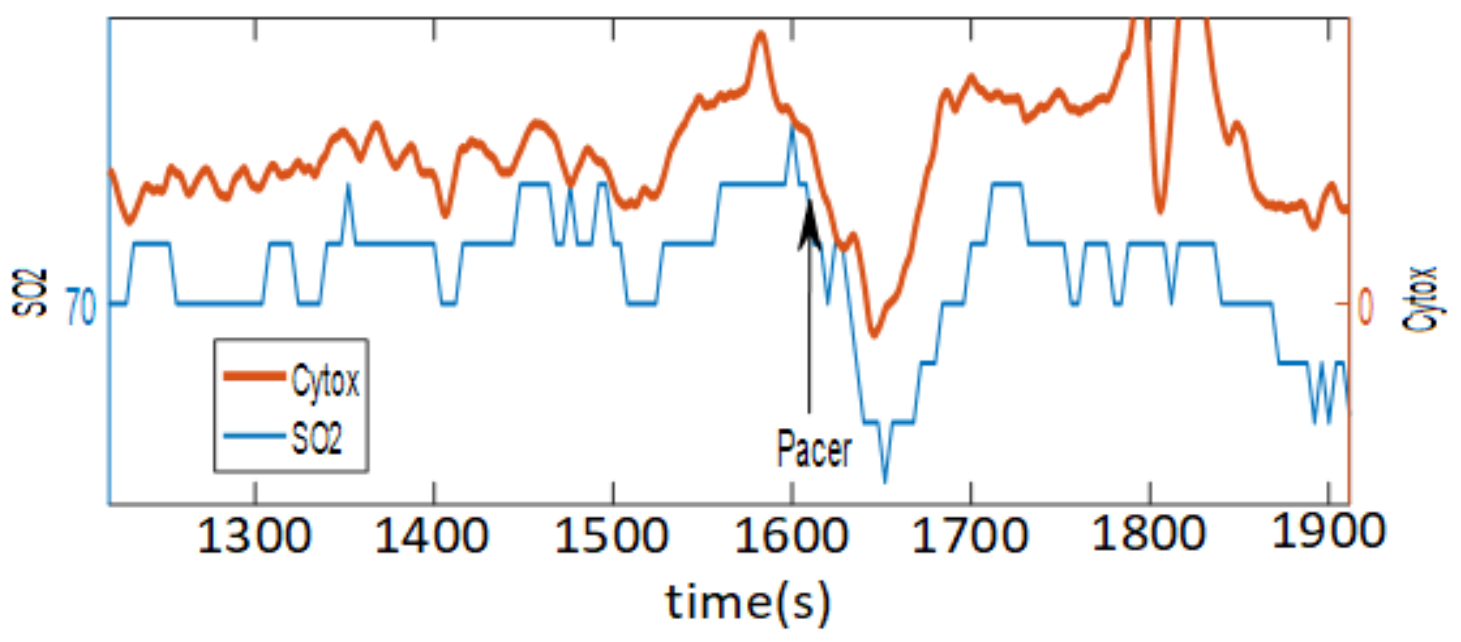

Figure 3.19 Patient 2: comparison of SO2 measured by mNIRS with Cyt-ox measured by hNIRS.

Figures 3.17. and 3.18. show plots of comparison for patient 1. Figure 3.17. compares the oxygen saturations as measured by hNIRS versus mNIRS (Nonin). This figure shows a clear consistency in the lines of the two methods and a significant drop during the introduction of the valve at around 2600 seconds. Figure 3.18. shows the comparison of SO2 versus Cyt-ox as measured by mNIRS and hNIRS respectively. It is observed that, although there is a significant drop in the $\mathrm{SO} 2$ when the pacer is on, there is no significant change in oxygen consumption. Figure 3.19. shows similar comparison as Figure 3.18. for patient 2. Although the SO2 behaves like that of patient 1, the hNIRS measurement shows a significant change in the oxygen consumption. And this is observed by a drop in both SO2 and Cyt-ox lines during the pacer introduction.

\section{5 fNIRS/fMRI Measurements}

This experiment was performed by a simultaneous measurement of fNIRS and fMRI to validate the results of the fNIRS with that obtained from the fMRI. The experimental set up 
is shown in Figure 3.20 below. In this set up we used the same spectrometers that were used during multichannel measurements and their specifications are indicated in section 2.2 above.

The fibers that were used to transmit light to the patient and collect data are home-made in our lab with the same specifications as the multichannel measurements. The lengths of these fiber bundles is about $6 \mathrm{~m}$ as the MRI room cannot have metal equipments inside of it and our measurements have to be done from the outside of the room. The probe used to hold the source and detector fiber bundles is made of a rubber band and is put around the head of the subjects. The source-detector distance was at around $3 \mathrm{~cm}$ for both spectrometers.
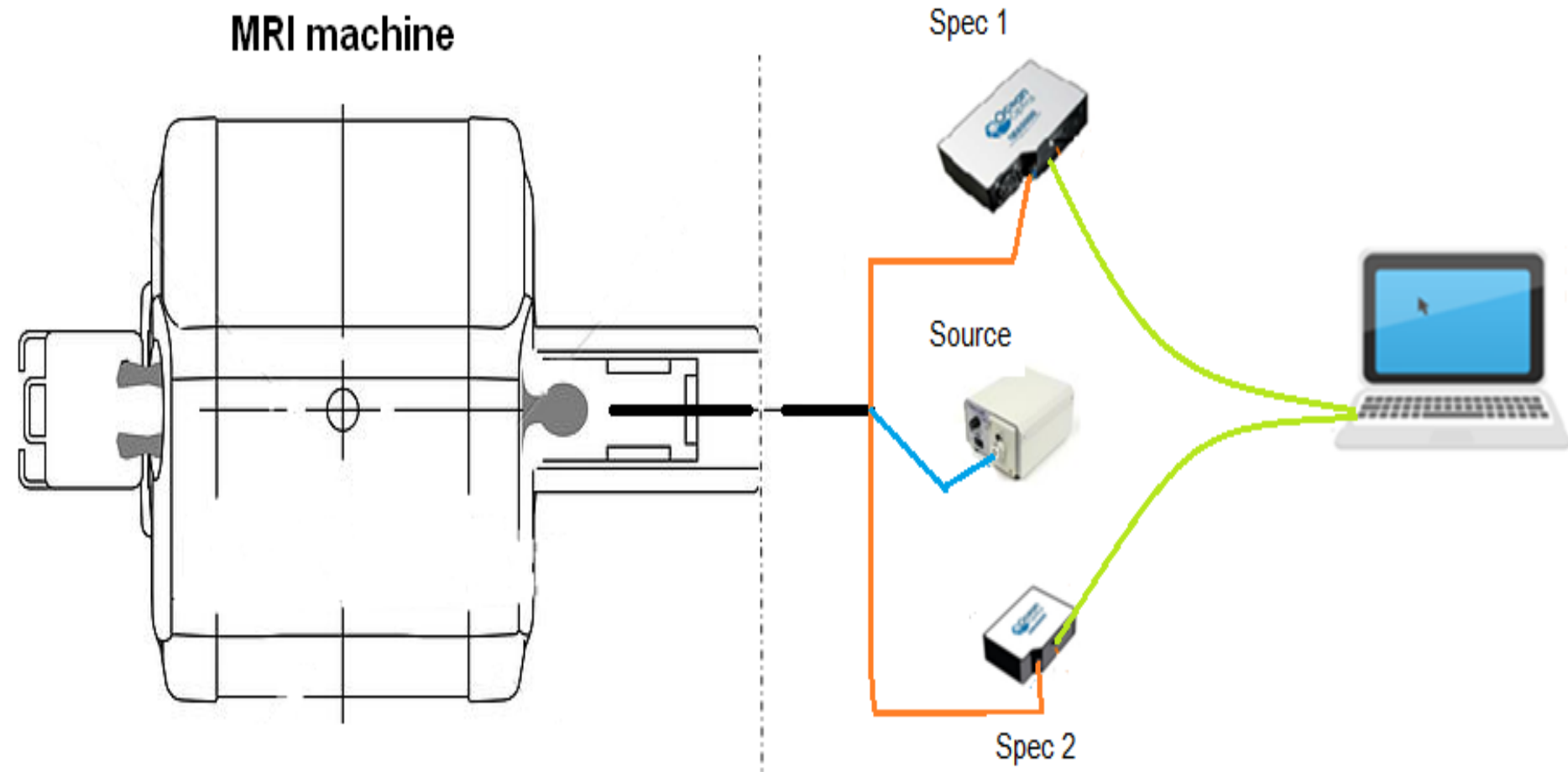

Figure 3.20 The experimental set up for an fNIRS/fMRI simultaneous measurement. The dashed line shows the separating wall between the MRI machine room and the adjacent room. 


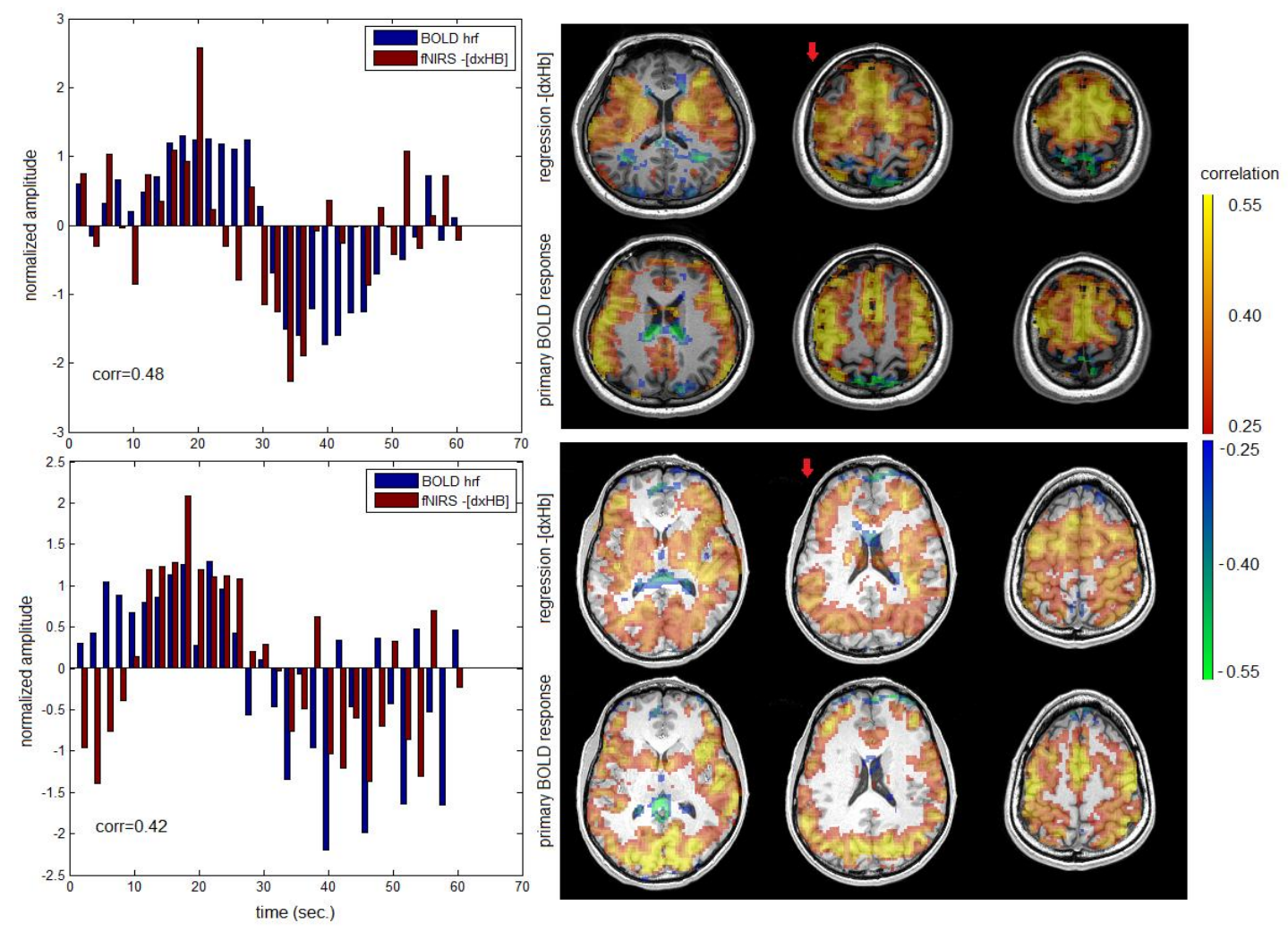

Figure 3.21 fNIRS Vs Primary BOLD HRF.

During the fMRI + hNIRS acquisition the cerebral hemodynamic changes as measured by fNIRS were compared with BOLD fMRI signals acquired simultaneously during breath hold regime. Figure 3.21 shows the time course of the primary BOLD HRF during the task epoch next to the $-[\mathrm{HHb}]$ time series (time series of the negative of deoxyhemoglobin concentration change) and their correlation. The plots on the right of Figure 3.21 shows a regression map (shown as correlation with $-[\mathrm{Hb}]$ ). This is the regression of the fNIRS $-[\mathrm{Hb}]$ against the fMRI average BOLD signal for each brain pixel. The red arrow points to the approximate location of the optode. 
The result obtained by the simultaneous measurement by fMRI and hNIRS during trail making is shown in Figure 3.22. below. We can observe that the trail making tasks (Trail A and B) activating the posterior brain regions.

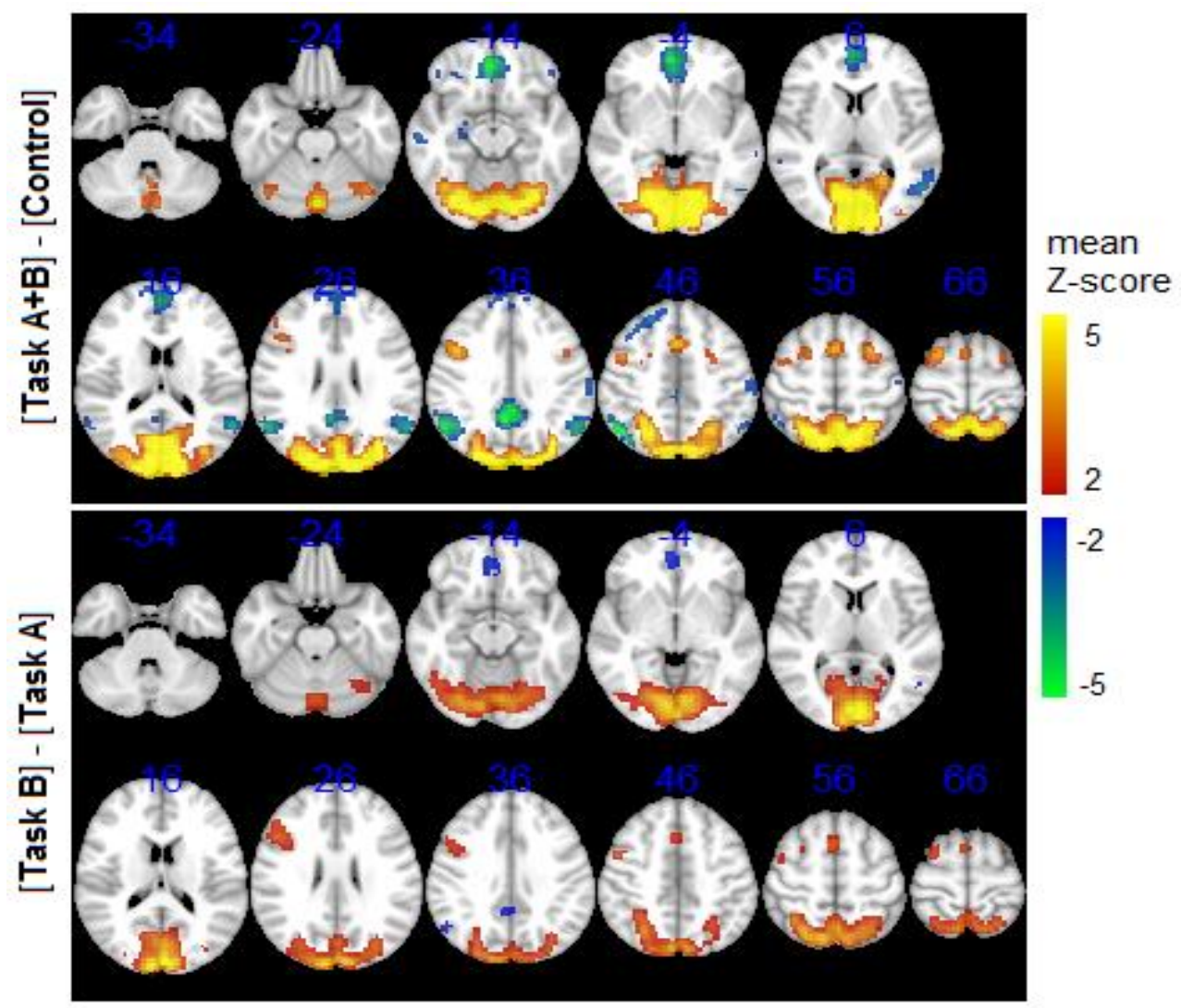

Figure 3.22 Mean Z-scored BOLD activation maps for trail making task, comparing Task A+B vs Control and Task A vs Task B 


\section{DISCUSSION}

In the current study we measured noninvasively cerebral parameters of different human subjects using hNIRS. In some measurements we compared hNIRS results with fMRI measurements. We had also the chance to measure the hNIRS parameters of cardiac patients with the TAVI procedures in the operating room. To accomplish these tasks, we needed to develop reliable equipment and methods. Also certain modifications have to be made to accommodate for specific factors of the MRI scanner and the hospital operating room. One of the requirements during these experiments was the longer lengths of the optical fibers to avoid interferences with the MRI machine and the movement of the operating team during TAVI. To achieve this we developed a fiber system that gives a stronger signal and to examine/test this we did multichannel hNIRS measurements that included tests on subjects to assess the brain response to cognitive tasks.

The developed fibers were tested for their efficiency and as Figure 2.2. indicates the spectra for the $2 \mathrm{~m}$ and $6.5 \mathrm{~m}$ fibers show the usual fNIRS spectrum shape. The improvements of the spectral intensity were significant for both lengths of fibers compared to the commercial analogs. These new fiber bundles were used to create headpieces fitting the forehead of the patient and leave no gaps that allow the loss of photons.

We also have shown the feasibility of the hNIRS multichannel measurements, which is necessary in functional brain measurements. As can be observed from the SNR of data recorded at different integration times (Figure 2.7.) shows that data can be acquired at faster rate during multichannel measurement when the integration time is $100 \mathrm{~ms}$. This is very acute during some clinical procedures as the events that are measured can be very short and the 
procedures are fast-paced. Being able to do multichannel experiments has the advantage of acquiring data from several regions of the head and that can help in advancing the analysis of the data.

During the multichannel experiment, our hNIRS system was tested on eleven control subjects to acquire the cerebral blood oxygenation during neurocognitive tasks of Eyes Open/Close and Trail Making. Unfortunately, during these functional measurements we did not observe statistically-significant task-related changes by hNIRS. This can be explained by the fact we later learned from fMRI measurements, that the brain activation paradigms used in our experiments primarily activate the occipital regions and not the pre-frontal cortex where the hNIRS probes were located. Previously, Cooper et al [37] have shown that Cyt-ox redox changes can be measured non-invasively with hNIRS (single-channel). Cyt-ox redox changes are very important because the mark the intracellular oxygen consumption unlike hemoglobin, which is related to blood flow changes. Cyt-ox redox changes can be monitored by hNIRS as the copper center of the Cyt-ox has a distinct redox-sensitive absorption spectrum (a decrease in copper concentration gives a diminished NIR absorbance) in the near-infrared region [37].

In our experiment of the occipital region, we recorded brain activity during the neurocognitive tasks of Eyes Open/Close. The difficulty of measuring the occipital signals by fNIRS is that dense hair with black pigment strongly absorbs light. Our custom made probe enabled us to get a statistically significant results for light haired subjects especially for $\mathrm{CCO}$ (which is usually hard to obtain) and $\mathrm{SO} 2$. 
During the fMRI/fNIRS measurements we were able to measure consistent brain responses to breath-holding. Both fMRI and fNIRS recorded consistent blood oxygenation changes. We observed a good correlation between hNIRS and fMRI measurements which was indicated by concurrent opposite changes in the fMRI BOLD signal and $[\mathrm{Hb}]$ measured by hNIRS in the activated areas of the brain in comparison with the resting state. The fNIRS time course and brain pattern in Figure 3.21. indicate that the negative of $[\mathrm{Hb}]$ change was well consistent with the BOLD HRF signal (corr =0.48). This consistency was repeated with the second subject having a strong correlation (corr $=0.42$ ) between the fNIRS and BOLD HRF.

The results from our experiments during cardiac surgery indicated that a reduced brain activation of the patients was recorded using fNIRS. This was indicated in Figure 3.14. and 3.15. as a drop in the graph when the pacer is put on. In Figure 3.15., the pacer was on at 3600 seconds, 4000 seconds and 4300 seconds. During this time, when the pacer is put on, the heart is momentarily stopped and this is recorded as a drop in the spectra. Both the $\mathrm{Hb}$ and $\mathrm{HbO} 2$ concentrations are seen to be dropping during these instances. A similar phenomenon is also shown in Figure 3.14. for the oxygen saturation (SO2). In this study our result using fNIRS indicated a reduced cerebral SO2 in all patients during pacing, which is in contrast with findings of the Nonin measurements. This was indicated as a significant drop in the curves during pacing three times in Figure 3.16. Results from patient 2, although not replicated on patient 1 , also indicate a significant Cyt-ox reduction as measured by hNIRS during a moment when the pacer is on which was consistent with that of SO2 measured by mNIRS. 


\section{CONCLUSIONS}

In this thesis I presented the development of the hyperspectral system for near-infrared spectroscopy of tissues including multi-channel setup and novel fiber-optic optodes optimized for hNIRS and demonstrated the suitability of the system in several clinical applications. In conclusion:

- Our home-made fiber bundles gave a significant signal improvement compared to the commercial fibers. This increase in signal was highlighted in the acquisition of Cyt-ox responses that is usually difficult to obtain. And longer fiber bundles were made that fit clinical settings.

- The novel multichannel set up allowed us to measure four sites on the human brain acquiring data with acceptable sampling rate and signal-to-noise ratio, simultaneously with the wireless EEG system (Emotiv, USA). These measurements indicated that Cyt-ox shows clearer response to brain stimuli than hemodynamic measures. This can be because blood is present in all tissue layers, while Cytochrome is mainly in the brain.

- During the simultaneous fMRI/hNIRS measurements carried out with our homemade fibres the hNIRS was of good quality and well correlated with fMRI BOLD signal during breath holding in multiple human subjects. This confirmed that our new fibre design was advantageous for measurements where very long fibres were required.

- During the TAVI surgery on cardiac patients our measurements by hNIRS provided valid and important brain monitoring data streams. While hNIRS 
provided measurements of cerebral tissue oxygen saturation close to those obtained using a commercial mNIRS system, in addition to that hNIRS allowed to measure separately oxy- and deoxy-hemoglobin, the total blood volume, and redox changes in cytochrome $\mathrm{C}$, thus providing the direct assessment of oxygen metabolism in cerebral neurons.

- We used our specially designed brush-like fibers that provided an increased penetration of light through human head hair, since the standard commercial fibers available were not designed for measurements of hairy parts of the head. We used these fibers during occipital measurements to probe the posterior lobe which is responsible for visual attention. Unfortunately we found that black hair posed a problem for hNIRS. This indicated that to increase the signal intensity without burning the tissue on hairy areas one should use narrow-band light sources such as laser diodes or light emitting diodes. 


\section{FUTURE WORK}

- In the future our home-made Fiber Optic Bundle and the multichannel setup can be used in hyperspectral fNIRS measurements for different cognitive tasks which can activate the pre-frontal cortex.

- hfNIRS should be tested to monitor brain activities during cognitive performances following concussion.

- The system in this study can be used to assess and analyze these changes in brain activity for comparison with fMRI measurements.

- Validation of hNIRS to measure cerebral oxygen consumption in cardiac patients. 


\section{REFERENCES}

[1] Davies, D. J., Su, Z., Clancy, M. T., Lucas,S. J. E., Dehghani, H., Logan, A., Belli, A. (2015) , Near-Infrared Spectroscopy in the Monitoring of Adult Traumatic Brain Injury: A Review. Journal of Neurotrauma. 32(13): 933 - 941.

[2] Arridge, S.R., Schweiger, M., Hiraoka, M., and Delpy, D.T. "A finite element approach for modeling photon transport in tissue," Am. Assoc. Phys. Med., Vol. 20, No. 2, 1993, pp. $299-309$.

[3] Jöbsis, F. F. "Noninvasive, Infrared Monitoring of Cerebral and Myocardial Oxygen Sufficiency and Circulatory Parameters," Science, Vol. 198, 4323, 1977, pp. $1264-1267$

[4] Bakker, A., Smith, B., Ainslie, P., and Smith, K. "Near-Infrared Spectroscopy, Applied Aspects of Ultrasonography in Humans," Prof Philip Ainslie (Ed), ISBN: 978-953-51-0522-0, InTech, Available from: http://www.intechopen.com/books/applied-aspects-of-ultrasonography-inhumans/near-infrared-spectroscopy

[5] Toronov, V., Webb, A., and Choi, J. H. "Investigation of human brain hemodynamics by simultaneous near-infrared spectroscopy and functional magnetic resonance imaging," Am. Assoc. Phys. Med., Vol. 28, No. 4, 2001, pp. $521-527$

[6] Matcher, S. J., Elwell, C. E., Cooper, C. E., Cope, M., Delpy, D. T., "Performance comparison of several published tissue near---infrared spectroscopy algorithms," Anal. Biochem., vol. 227(1), pp. 54-68, 1995.

[7] Yeganeh, H. Z., Toronov, V., Elliott, J. T., Diop, M., Lee, T. Y., and St. Lawrence, K. "Broadband continuous-wave technique to measure baseline values 
and changes in the tissue chromophore concentrations," Biomed. Opt Express, Vol. 3, no. 11, 2012, Pp. $2761-2770$.

[8] Pucci O., Toronov V., St Lawrence K., "Measurement of the optical properties of a two-layer model of the human head using broadband near-infrared spectroscopy,” Appl. Opt. 49(32), 6324-6332 (2010)

[9] Diop M., Elliott J. T., Tichauer K. M., Lee T.-Y., St Lawrence K., “A broadband continuous-wave multichannel near-infrared system for measuring regional cerebral blood flow and oxygen consumption in newborn piglets," Rev. Sci. Instrum. 80(5), 054302 (2009).

[10] Murkin, M., and Arango, M. "Near-infrared spectroscopy as an index of brain and tissue oxygenation," British Journal of Anaesthesia, Vol. 103, no. 1, Pp. i3 - i13, 2009.

[11] Wahr, J. A., Tremper, K. K., Samra, S., and Delpy, D. T. "Near-Infrared Spectroscopy: Theory and Applications," Journal of Cardiothoracic and vascular Anesthesia, Vol. 10, no 3, 1996 pp 406 - 418.

[12] Izzetoglu, M., Izzetoglu, K., Bunce, S., Ayaz, H., Devaraj, A., Onaral, B., and Pourrezaei, K. "Functional Near-Infrared Neuroimaging," IEEE Transactions on Neural Systems and Rehabilitation Engineering, Vol. 13, no. 2, 2005, Pp. 153 159.

[13] Son, I., Yazici, B., "Near-Infrared Imaging and spectroscopy for Brain Activity Monitoring, Advances in Sensing with Security Applications," Jim Byrnes and Gerald Ostheimer (Ed), Pp. 341 - 372, 2006.

[14] Hoshi, Y. "Functional near-infrared optical imaging: Utility and limitations in human brain mapping," Society for Psychophysiological Research, Vol. 40, 2003, pp. $511-520$ 
[15] Strangman, G., Boas, D.A., and Sutton, J.P. "Non-Invasive Neuroimaging using Near-Infrared Light,” Biological Psychiatry, Vol. 52, 2002, Pp. 679 - 693.

[16] Sauer, M. (2011). "Basic principles of Fluorescence spectroscopy." Wiley-VCH Verlag GmbH \& Co.

[17] Villota, E.D., Carmona, M.T.G., Rubio, J.J., and Andres, S.R. "Equality of the in Vivo and in Vitro Oxygen-Binding Capacity of Haemoglobin in Patients with Severe Respiratory Disease," British Journal of Anaesthesia, Vol. 53, 1981, Pp. $1325-1328$.

[18] Villringer, A., and Chance, B. "Non-invasive optical spectroscopy and imaging of human brain function," Trends in Neurosciences, Vol. 20, no. 10, 1997, Pp. 435 442.

[19] Knowles JR (1980). "Enzyme-catalyzed phosphoryl transfer reactions". Annu. Rev. Biochem. 49: 877-919

[20] Karp G., Cell and Molecular Biology.: John Wiley \& Sons, 2008.

[21] Arridge, S.R., Schweiger, M., Hiraoka, M., and Delpy, D.T. "A finite element approach for modeling photon transport in tissue, "Am. Assoc. Phys. Med., Vol. 20, no. 2, 1993 Pp. $299-309$.

[22] Obrig, H., and Villringer, A. "Beyond the Visible - Imaging the Human Brain With Light," Journal of Cerebral Blood Flow \& Metabolism, Vol. 23, no. 1, 2003, Pp. $1-18$.

[23] Elwell, C., and Hebden, J. "Near Infrared Spectroscopy," Biomedical Optics Research Laboratory. Available from http://www.ucl.ac.uk/medphys/research/borl/intro/nirs

[24] Ogawa, S., Menon, R.S., Tank, D.W., Kim, S.G., Merkle, H., Ellermann, J.M.,and Ugurbil, K. "Functional brain mapping by blood oxygenation level-dependent contrast magnetic resonance imaging: A comparison of signal characteristics with a biophysical model,” Biophys Journal, Vol. 64, 1993, Pp. 803 - 812. 
[25] Wang, L. V. and Wu, H. Biomedical Optics - Principles and Imaging. WileyInterscience, 2007.

[26] Delpy, D.T., Cope, M., van der Zee, P., Arridge, S., Wray, S., and Wyatt, J. "Estimation of optical pathlength through tissue from direct time of flight measurement," Phys. Med. Biol., Vol. 33, No. 12, 1988, Pp. 1433 - 1442.

[27] Arridge, S.R., Schweiger, M., Hiraoka, M., and Delpy, D.T. "A finite element approach for modeling photon transport in tissue, “Am. Assoc. Phys. Med., Vol. 20, no. 2, 1993 Pp. $299-309$.

[28] Kwong, K.K., Belliveau, L.W., chesler, D.A., Goldberg, I.E., Weisskoff, R.M., Poncelet, B.P., Kennedy, D.N., Hoppel, B.E., Cohen, M.S., and Turner, R. "Dynamic magnetic resonance imaging of human brain activity during primary sensory stimulation," Proc. Natl. Acad. Sci. USA, Vol. 89, no. 12, 1992, Pp. 5675 -5679 .

[29] Ogawa, S., Lee, T. M., Kay, A.R., and Tank, D. W. "Brain magnetic resonance imaging with contrast dependent on blood oxygenation," Proc. Natl. Acad. Sci. USA, Vol. 87, 1990, pp. 9868 - 9872.

[30] I Schelkanova and V Toronov, "Independent component analysis of broadband 84 near---infrared spectroscopy data acquired on adult human head," Biomed Opt Express, vol. 3, pp. 64---74, 2012.

[31] Gratton, E., Toronov, V., Wolf, U., Wolf, M., and Webb, A. "Measurement of brain activity by near-infrared light," Journal of Biomedical Optics, Vol. 10, no. 1, 2005. Available from: http://dx.doi.org/10.1117/1.1854673

[32] http://spinwarp.ucsd.edu/neuroweb/Text/br-100.htm

[33] Gratton, E., Toronov, V., Wolf, U., Wolf, M., and Webb, A. "Measurement of brain activity by near-infrared light," Journal of Biomedical Optics, Vol. 10, no. 1, 2005. Available from: http://dx.doi.org/10.1117/1.1854673 
[34] Mehagnoul-Shipper, D. J., van der Kallen, B.F., Colier, W.N., van der Sluijs, M.C., van Erning, L.J., Thijssen, H.O., Qesenburg, B., Hoefnagels, W.H., and Jansen, R.W. "Simultaneous measurements of cerebral changes during brain activation by near-infrared spectroscopy and functional magnetic resonance imaging in healthy young and elderly subjects," Hum Brain Mapp., Vol. 16, no. 1, 2002, Pp. $14-23$.

[35] Kandel, E., Schwartz, J. and Jessell, T. Principles of Neural Science. $3^{\text {rd }}$ Ed. New York: N.Y. Elsevier, 1991.

[36] TAVI, Transcatheter aortic valve implantation, Ottawa Heart Institute. http://www.ottawaheart.ca/test-procedure/tavi-transcatheter-aortic-valve$\underline{\text { implantation }}$

[37] Cooper, C. E. et al. Use of mitochondrial inhibitors to demonstrate that cytochrome oxidase near-infrared spectroscopy can measure mitochondrial dysfunction noninvasively in the brain. J. Cereb. Blood Flow Metab. 19, 27-38 (1999).

[38] Son, Il-Y. and Yazici, B. "Near infrared imaging and spectroscopy for brain activity monitoring," NATO Security through Science Series A: Chemistry and Biology, pp. 341-372, 2006

[39] Reich, G. "Near-infrared spectroscopy and imaging: Basic principles and pharmaceutical applications," Advanced Drug Delivery Reviews, Vol. 57, no. 8, 2005, Pp. 1109 - 1143.

[40] Kocsis, L., Herman, P. and Eke, A. "The modified Beer-Lambert law revisited," Phys. Med. Biol., Vol. 51, No. 91, 2006. 